\title{
REGULARIZATIONS OF RESIDUE CURRENTS
}

\author{
JAN-ERIK BJÖRK \& HÅKAN SAMUELSSON
}

\begin{abstract}
Under assumptions about complete intersection, we prove that Coleff-Herrera type currents satisfy a robust calculus in the sense that natural regularizations of such currents can be multiplied to yield regularizations of the Coleff-Herrera product of the currents.
\end{abstract}

\section{INTRODUCTION}

Let $f$ be a holomorphic function defined on the unit ball $\mathbb{B} \subset \mathbb{C}^{n}$. Then $1 / f$ exists as a principal value distribution, or rather as a $(0,0)$ current, on $\mathbb{B}$, i.e.,

$$
\lim _{\epsilon \rightarrow 0^{+}} \int_{\{|f|>\epsilon\}} \varphi / f
$$

exists for $\varphi \in \mathscr{D}_{n, n}(\mathbb{B})$ and defines a continuous functional on $\mathscr{D}_{n, n}(\mathbb{B})$. This was first proved by Herrera-Lieberman, [17, using Hironaka's theorem on resolutions of singularities. In fact, by Hironaka's theorem one may assume that $f$ is a monomial, and in that case it is possible to compute the limit by hand. The proof also shows that one may take the limit of integrals over $\{|\tilde{f}|>\epsilon\}$, where $\tilde{f}$ is any holomorphic function such that $\tilde{f}^{-1}(0) \supseteq f^{-1}(0)$. The current $1 / f$ is obviously closely related to division problems; if $h$ is holomorphic then $h / f$ is at least a current, and it is holomorphic if and only if it is $\bar{\partial}$-closed, i.e., if and only if $0=\bar{\partial}(h / f)=h \bar{\partial}(1 / f)$. Hence, $h$ is in the ideal, $\langle f\rangle$, generated by $f$ if and only if $h$ annihilates the current $\bar{\partial}(1 / f)$. This current clearly has support on $Z_{f}=f^{-1}(0)$ and it is related to Lelong's integration current $\left[Z_{f}\right]$, see [20], by the Poincarè-Lelong formula: $2 \pi i\left[Z_{f}\right]=\bar{\partial}(1 / f) \wedge d f$. The current $\bar{\partial}(1 / f)$ is called the residue current associated to $f$ and it is thus an analytic object that describes the algebraic-geometric object $\langle f\rangle$.

Now, let $V$ be a pure $n$-dimensional analytic subset of a complex $N$ dimensional manifold $X$ and let $f: X \rightarrow \mathbb{C}$ be a holomorphic function such that $V \backslash f^{-1}(0)$ is a dense subset of $V_{\text {reg }}$. Then the principal value

The second author was partially supported by a Post Doctoral Fellowship from the Swedish Research Council. 
of $1 / f$ exists on $V$, i.e.,

$$
\lim _{\epsilon \rightarrow 0^{+}} \int_{V \cap\{|\tilde{f}|>\epsilon\}} \varphi / f
$$

exists for $\varphi \in \mathscr{D}_{n, n}(X)$ and holomorphic $\tilde{f}$ with $\tilde{f}^{-1}(0) \supseteq f^{-1}(0)$ and yields a well defined current denoted $(1 / f)[V]$. The existence of this limit follows from the case $V=\mathbb{B}$ by Hironaka's theorem. A sheaf of currents on $X$ supported on $V$ is then obtained by applying holomorphic differential operators to such currents. This sheaf is (equivalent to) the sheaf $\mathbf{C H}_{V}[* S]$, see Definition 3, and it is this kind of currents we will consider in this paper. The kernel of $\bar{\partial}$ in $\mathbf{C H}_{V}[* S]$ is denoted $\mathbf{C H}_{V}$ and is actually sufficiently ample to represent moderate cohomology in the sense that $\mathbf{C H}_{V} \simeq \mathcal{H}_{[V]}^{P}\left(\mathcal{O}_{X}\right)$, see [14]; here $P=N-n$ is the codimension of $V$. The notation $\mathbf{C H}$ refers to Coleff-Herrera type currents.

Let us return to the case $V=\mathbb{B} \subset \mathbb{C}^{n}$ and consider a holomorphic mapping $f=\left(f_{1}, \ldots, f_{p}\right): \mathbb{B} \rightarrow \mathbb{C}^{p}$. To find a current that describes the ideal, $\langle f\rangle$, generated by $f_{1}, \ldots, f_{p}$ it is tempting to try to define the product $\bar{\partial}\left(1 / f_{1}\right) \wedge \cdots \wedge \bar{\partial}\left(1 / f_{p}\right)$. If $f$ defines a complete intersection, i.e., $f^{-1}(0)$ has codimension $p$, it is possible to give a well defined meaning to this product. This was first done by Coleff-Herrera, [13], as follows. Let $\varphi \in \mathscr{D}_{n, n-p}(\mathbb{B})$ and put

$$
I_{f}^{\varphi}(\epsilon):=\int_{\cap\left\{\left.f_{j}\right|^{2}=\epsilon_{j}\right\}} \varphi /\left(f_{1} \cdots f_{p}\right) .
$$

Coleff-Herrera proved that the limit of $I_{f}^{\varphi}(\epsilon)$ as $\epsilon \rightarrow 0$ along any "admissible path" exists and defines a current of bidegree $(0, p)$, denoted $\bar{\partial}\left(1 / f_{1}\right) \wedge \cdots \wedge \bar{\partial}\left(1 / f_{p}\right)$ or $R^{f}$ for short, that is alternating with respect to the ordering of the tuple $f$. Admissible path here means that $\epsilon \rightarrow 0$ along a path in the first orthant such that $\epsilon_{j} / \epsilon_{j-1}^{k} \rightarrow 0$ for $j=2, \ldots, p$ and all $k \in \mathbb{N}$. It was later proved by Dickenstein-Sessa, [14, and Passare, [21], independently that $R^{f}$ describes the ideal $\langle f\rangle$ in the sense that its annihilator is precisely $\langle f\rangle$. We remark that even if $f$ does not define a complete intersection, the limit of $I_{f}^{\varphi}(\epsilon)$ along an admissible path exists but does not yield a well defined current associated to $f$ as one easily sees from the simple case $f_{1}=z^{2}, f_{2}=z w$. Currents describing general ideals have recently been defined by Andersson-Wulcan, [6]; see also Section 6 below.

From now on we stick to the (generic) case that $f$ defines a complete intersection. The first question raised by Coleff-Herrera in 13] is whether it is necessary to take limits along admissible paths or not. It turned out to be necessary; Passare-Tsikh, [24], showed that if $f=\left(z^{4}, z^{2}+w^{2}+z^{3}\right)$ then $I_{f}^{\varphi}(\epsilon)$ does not have an unrestricted limit as $\epsilon \rightarrow 0$ (for all $\varphi$ ). A generic family of examples with this property was 
later found by the first author; even examples with $I_{f}^{\varphi}(\epsilon) \rightarrow \infty$ along certain paths are constructed, see, e.g., [12]; see also Pavlova, 26]. However, our main theorem implies that the mild average of $I_{f}^{\varphi}(\epsilon)$,

$$
\mathcal{I}_{f}^{\varphi}(\epsilon):=\int_{s \in[0, \infty)^{p}} I_{f}^{\varphi}(s) d \chi_{1}\left(s_{1} / \epsilon_{1}\right) \wedge \cdots \wedge d \chi_{p}\left(s_{p} / \epsilon_{p}\right),
$$

where $\chi_{j} \in C^{\infty}([0, \infty]), \chi_{j}(0)=0$, and $\chi_{j}(\infty)=1$, depends Hölder continuously on $\epsilon \in[0, \infty)^{p}$ and tends to the Coleff-Herrera product $R^{f}$ as $\epsilon \rightarrow 0$. In fact, we prove

Theorem 1. Let $X$ be a complex $N$-dimensional manifold, $V \subseteq X$ an analytic subset of pure dimension $n$, and $f=\left(f_{1}, \ldots, f_{q}\right): X \rightarrow$ $\mathbb{C}^{q}$ a holomorphic mapping such that $\left(f_{1}, \ldots, f_{p}, f_{j}\right)$ locally defines a complete intersection on $V$ for $p+1 \leq j \leq q$. Let also $\chi_{j}, 1 \leq j \leq q$, be smooth on $[0, \infty]$, vanish to order $\ell_{j}$ at 0 and $\chi_{j}(\infty)=1$. Then for any $\mu \in \mathbf{C H}_{V}$ and $\varphi \in \mathscr{D}_{N, n-p}(X)$ we have

$$
\begin{aligned}
& \mid \frac{\bar{\partial} \chi_{1}^{\epsilon} \wedge \cdots \wedge \bar{\partial} \chi_{p}^{\epsilon} \chi_{p+1}^{\epsilon} \cdots \chi_{q}^{\epsilon}}{f_{1}^{\ell_{1}} \cdots f_{q}^{\ell_{q}}} \wedge \mu . \varphi \\
& \quad-\bar{\partial} \frac{1}{f_{1}^{\ell_{1}}} \wedge \cdots \wedge \bar{\partial} \frac{1}{f_{p}^{\ell_{p}}} \frac{1}{f_{p+1}^{\ell_{p+1}} \cdots f_{q}^{\ell_{q}}} \wedge \mu . \varphi \mid \leq C\|\varphi\|_{M} \epsilon^{\omega},
\end{aligned}
$$

where $\chi_{j}^{\epsilon}=\chi_{j}\left(\left|f_{j}\right|^{2} / \epsilon_{j}\right)$, and $M$ and $\omega$ are positive constants that only depend on $f$ and $\operatorname{Supp}(\varphi)$, while the positive constant $C$ also might depend on the $C^{M}$-norm of the $\chi_{j}$-functions.

Let $V=X, \mu=1, \ell_{j}=1$ and let the $\chi_{j}$ be smooth regularizations of the characteristic function of $[1, \infty)$; by this we mean that $\chi_{j}$ is a smooth increasing function on $[0, \infty)$ that is 0 close to 0 and 1 close to $\infty$. The theorem implies that the smooth form

$$
\bar{\partial} \frac{\chi_{1}^{\epsilon}}{f_{1}} \wedge \cdots \wedge \bar{\partial} \frac{\chi_{p}^{\epsilon}}{f_{p}} \cdot \frac{\chi_{p+1}^{\epsilon}}{f_{p+1}} \cdots \frac{\chi_{q}^{\epsilon}}{f_{q}}
$$

converges unrestrictedly to the mixed residue and principal value current

$$
\bar{\partial} \frac{1}{f_{1}} \wedge \cdots \wedge \bar{\partial} \frac{1}{f_{p}} \cdot \frac{1}{f_{p+1}} \cdots \frac{1}{f_{q}}
$$

introduced by Coleff-Herrera, [13], and Passare, 22]. It is proved in [22] that if $\epsilon_{j}=\delta^{s_{j}}$, then (2) has a limit, independent of $s=\left(s_{1}, \ldots, s_{q}\right) \in$ $\mathbb{R}_{+}^{q}$, as $\delta \rightarrow 0^{+}$as long as $s$ avoids finitely many hyperplanes $H_{a}=\{t \in$ $\left.\mathbb{R}_{+}^{q} ; t \cdot a=0\right\}, a \in \mathbb{N}^{q}$; we say that $\epsilon \rightarrow 0$ inside a Passare sector. Our result is thus a sharpening and a generalization of Passare's result and shows that there is a robust calculus for Coleff-Herrera type currents. 
In particular, we have the appealing formula

$$
R^{f} \cdot \varphi=\lim _{\epsilon \rightarrow 0} \int_{X} \bar{\partial} \frac{\bar{f}_{1}}{\left|f_{1}\right|^{2}+\epsilon_{1}} \wedge \cdots \wedge \bar{\partial} \frac{\bar{f}_{p}}{\left|f_{p}\right|^{2}+\epsilon_{p}} \wedge \varphi, \quad \varphi \in \mathscr{D}_{N, N-p}(X),
$$

which follows by taking $\chi_{j}(t)=t /(t+1)$.

Another approach to the Coleff-Herrera product, $R^{f}$, is based on analytic continuation of currents, a technique with roots in the works of Atiyah, [8], and Gelfand-Shilov, [16]. In the context of residue currents, it has been developed by several authors, e.g., Barlet-Maire, 9], Yger, [29], Passare-Tsikh, [23], Berenstein-Gay-Yger, [11], and by the second author in the recent paper [28]. Computing the Mellin transform of the integral in (1) (considered as a function of $\epsilon$ ) one obtains

$$
\int_{V}|\tilde{f}|^{2 \lambda} \varphi / f
$$

if $\mathfrak{R e} \lambda>>1$. One can show, either by using a Bernstein-Sato functional equation or by computing directly in a resolution of $V$ where $f^{-1}(0)$ has normal crossings, that (4) (as a function of $\lambda$ ) has a meromorphic continuation to all of $\mathbb{C}$ and that its poles are contained in an arithmetic progression $\{-s-\mathbb{N}\}, s \in \mathbb{Q}_{+}$. It is thus analytic in a neighborhood of the origin, and moreover, its value there defines the action of a current. This current is the current $(1 / f)[V]$, as one easily shows in a resolution.

The Coleff-Herrera-Passare current (3) can be obtained in a similar manner; consider the function

$$
\lambda \mapsto \int_{X} \frac{\bar{\partial}\left|f_{1}\right|^{2 \lambda_{1}} \wedge \cdots \wedge \bar{\partial}\left|f_{p}\right|^{2 \lambda_{p}}\left|f_{p+1}\right|^{2 \lambda_{p+1}} \cdots\left|f_{q}\right|^{2 \lambda_{q}}}{f_{1} \cdots f_{q}} \wedge \varphi,
$$

where $f$ defines a complete intersection on $X$ and $\mathfrak{R e} \lambda_{j}>>1$. One can similarly show that it has a meromorphic extension to $\mathbb{C}^{q}$. It was recently showed by the second author in [28] that it actually is analytic in a neighborhood of $\cap_{j}\left\{\mathfrak{R e} \lambda_{j} \geq 0\right\}$. By results of Yger, it was known before that the restriction of (5) to any complex line of the form $\left\{\lambda=\left(t_{1} z, \ldots, t_{q} z\right) ; z \in \mathbb{C}\right\}, t_{j} \in \mathbb{R}_{+}$, has an analytic continuation to a neighborhood containing the origin and that the value there equals (31). Moreover, it was also known that if $q=2$, then (5) has an analytic continuation to a neighborhood of the origin in $\mathbb{C}^{2}$; see, e.g., [10], 11] for proofs. Event though not explicitly stated in [28], we remark that it follows from the proof that one may replace $X$ in (5) by a pure dimensional analytic subset $V$ of $X$ and still have analyticity in a neighborhood of the origin.

We conclude the introduction with the simple but useful observation that expressions like $\chi\left(|f|^{2} / \epsilon\right) / f^{\ell}$ essentially are invariant under holomorphic differential operators. More precisely, if $\chi \in C^{\infty}([0, \infty])$ and 
vanish to order $\ell$ at 0 , then

$$
\frac{\partial}{\partial z_{j}} \frac{\chi\left(|f|^{2} / \epsilon\right)}{f^{\ell}}=\frac{\partial f}{\partial z_{j}} \frac{\tilde{\chi}\left(|f|^{2} / \epsilon\right)}{f^{\ell+1}}
$$

where $\tilde{\chi}(t)=t \chi^{\prime}(t)-\ell \chi(t)$ is smooth on $[0, \infty]$, vanishes to order $\ell+1$ at 0 , and $\chi(\infty)=-\ell \chi(\infty)$.

\section{The CASE OF THREE FUNCTIONS}

We first note that $I_{f}^{\varphi}(\epsilon)$ might be discontinuous already when $f=$ $\left(f_{1}, f_{2}\right)$ consists of two functions as the Passare-Tsikh example shows. The technical reason is the presence of charts of resonance, i.e., charts on the resolution manifold where it is not possible to choose coordinates so that the pullback of both $f_{1}$ and $f_{2}$ are monomials. To deal with the charts of resonance the smoothness of the $\chi$-functions has to be used; we refer to [27] for the details. In the case of three functions a new difficulty arise; it is no longer a local problem on the resolution manifold to prove that

$$
\int \bar{\partial} \frac{\bar{f}_{1}}{\left|f_{1}\right|^{2}+\epsilon_{1}} \wedge \bar{\partial} \frac{\bar{f}_{2}}{\left|f_{2}\right|^{2}+\epsilon_{2}} \frac{\bar{f}_{3}}{\left|f_{3}\right|^{2}+\epsilon_{3}} \wedge \varphi
$$

has an unrestricted limit. We illustrate this by considering a simple example; the example of Section 3 in [28. We let $f_{1}=z_{1}, f_{2}=z_{2}$, and $f_{3}=z_{3}$ in $\mathbb{C}^{3}$. Then, obviously, (7) has an unrestricted limit for all $(3,1)$-test forms $\varphi$ in $\mathbb{C}^{3}$. Now, we let $\varphi=\phi d z \wedge d \bar{z}_{3}$, where $\phi$ is a test function, we blow up $\mathbb{C}^{3}$ along the $z_{3}$-axis, and we compute (7) on the blow-up. The blow-up has two standard charts, one of which is given by $\left(w_{1}, w_{2}, w_{3}\right) \mapsto\left(w_{1}, w_{1} w_{2}, w_{3}\right)=\left(z_{1}, z_{2}, z_{3}\right)$. Let us consider the contribution, $\mu^{\varphi}(\epsilon)$, to (7) from this chart. One verifies easily, using Cauchy's formula, that $\lim _{\epsilon_{1} \rightarrow 0^{+}} \mu^{\varphi}(\epsilon)=0$ for fixed $\epsilon_{2}, \epsilon_{3}>0$. On the other hand, one similarly shows that

$$
\lim _{\epsilon_{3} \rightarrow 0^{+}} \lim _{\epsilon_{1} \rightarrow 0^{+}} \lim _{\epsilon_{2} \rightarrow 0^{+}} \mu^{\varphi}(\epsilon)=-(2 \pi i)^{2} \int_{z_{3}} \frac{\phi\left(0,0, z_{3}\right)}{z_{3}} d z_{3} \wedge d \bar{z}_{3},
$$

which clearly is non-zero for certain choices of $\phi$. Both the charts on the blow-up therefore have to be considered in order to see that (7) has an unrestricted limit.

In general, however, what can be showed in each chart separately is that

$$
\left|\int \bar{\partial} \frac{\bar{f}_{1}}{\left|f_{1}\right|^{2}+\epsilon_{1}} \wedge \bar{\partial} \frac{\bar{f}_{2}}{\left|f_{2}\right|^{2}+\epsilon_{2}} \wedge\left(\frac{\bar{f}_{3}}{\left|f_{3}\right|^{2}+\epsilon_{3}}-\frac{1}{f_{3}}\right) \varphi\right| \leq C \epsilon_{3}^{\omega}
$$

for positive constants $C$ and $\omega$ that do not depend on $\epsilon_{1}, \epsilon_{2}$, see Proposition 8 below. To see that (7) has an unrestricted limit it is therefore 
enough to show that

$$
\begin{aligned}
& \int \bar{\partial} \frac{\bar{f}_{1}}{\left|f_{1}\right|^{2}+\epsilon_{1}} \wedge \bar{\partial} \frac{\bar{f}_{2}}{\left|f_{2}\right|^{2}+\epsilon_{2}} \wedge \frac{1}{f_{3}} \varphi=\int \bar{\partial} \frac{\bar{f}_{1}}{\left|f_{1}\right|^{2}+\epsilon_{1}} \frac{\bar{f}_{2}}{\left|f_{2}\right|^{2}+\epsilon_{2}} \frac{1}{f_{3}} \wedge \bar{\partial} \varphi \\
& +\int \bar{\partial} \frac{\bar{f}_{1}}{\left|f_{1}\right|^{2}+\epsilon_{1}} \frac{\bar{f}_{2}}{\left|f_{2}\right|^{2}+\epsilon_{2}} \wedge \bar{\partial} \frac{1}{f_{3}} \wedge \varphi
\end{aligned}
$$

has an unrestricted limit. But now we have only two parameters and, moreover, on the right hand side there is only $\bar{\partial}$ in front of one of the parameter depending factors. With an appropriate induction hypothesis and the result of Proposition 8 one can then conclude that (7) has an unrestricted limit; see Section 6 for details.

\section{ColefF-Herrera CURrents}

In this section we review the facts we will need about Coleff-Herrera type currents. The results are well-known but for the readers convenience we supply detailed proofs. Let $X$ be a complex $N$-dimensional manifold and let $V \subseteq X$ be a reduced subvariety of pure dimension $n$. Put $P=N-n$ and let $\mathcal{J}_{V}$ be the ideal (sheaf) generated by $V$. A (possibly singular) hypersurface $S \subset X$ is called $V$-polar if $V \backslash S$ is a dense subset of $V_{\text {reg. }}$. Recall from the introduction, cf. (11), that if $h \in \mathcal{O}(X)$ and $h^{-1}(0)$ is $V$-polar, then the principal value current $(1 / h)[V]$ exists. It is often convenient to use the technique of analytic continuation when working with this current; recall from the introduction that if $\tilde{h}$ is any holomorphic function such that $\tilde{h}^{-1}(0)$ is $V$-polar and contains $h^{-1}(0)$, then

$$
\frac{1}{h}[V] \cdot \varphi=\left.\int_{V}|\tilde{h}|^{2 \lambda} \frac{\varphi}{h}\right|_{\lambda=0}, \quad \varphi \in \mathscr{D}_{n, n}(X) .
$$

The next lemma shows, in particular, that this kind of currents have the Standard Extension Property; a current $\mu$ has the Standard Extension Property with respect to a pure dimensional analytic set $V$ if for any holomorphic function $g$ such that $V \backslash g^{-1}(0)$ is dense in $V$ we have $\lim _{\epsilon \rightarrow 0^{+}} \chi\left(|g|^{2} / \epsilon\right) \mu=\mu$, where $\chi$ is a smooth regularization of the characteristic function of $[1, \infty)$. Two currents, $\mu$ and $\tilde{\mu}$, which both have the Standard Extension Property and are equal outside a hypersurface $H$, i.e., $\mu . \varphi=\tilde{\mu} . \varphi$ if $\varphi$ has support outside $H$, are thus equal.

Lemma 2. Let $h, f \in \mathcal{O}(X)$ and assume that $h^{-1}(0)$ is $V$-polar and that $V \backslash f^{-1}(0)$ is dense in $V$. If $\chi$ is a bounded function on $[0, \infty]$ that is identically 0 close to 0 and continuous at $\infty$, then

$$
\lim _{\epsilon \rightarrow 0^{+}} \frac{\chi\left(|f|^{2} / \epsilon\right)}{f^{\ell}} \cdot \frac{1}{h}[V]=\frac{\chi(\infty)}{f^{\ell} h}[V] .
$$


Proof. By Hironaka's theorem one may assume that $V$ is an $n$-dimensional manifold and that $\{h \cdot f=0\}$ has normal crossings. Locally one can then choose coordinates $x$ such that $f=x^{\alpha}$ and $h=v x^{\beta}$, where $v$ is an invertible holomorphic function. Letting $\varphi=\phi d x \wedge d \bar{x}$ we thus see that $\chi /\left(f^{\ell} h\right)[V] . \varphi$ is a finite sum of terms like

$$
\left.\int_{V}\left|x^{\beta}\right|^{2 \lambda} \frac{\chi\left(\left|x^{\alpha}\right|^{2} / \epsilon\right)}{x^{\ell \alpha+\beta}} \frac{|v|^{2 \lambda}}{v} \phi d x \wedge d \bar{x}\right|_{\lambda=0} .
$$

By Lemma 6 in [27] we can write

$$
\frac{|v|^{2 \lambda}}{v} \phi=\sum_{K+L<\ell \alpha+\beta-1} x^{K} \bar{x}^{L} \Phi_{K, L}(\lambda, x)+\sum_{K+L=\ell \alpha+\beta-1} x^{K} \bar{x}^{L} \Phi_{K, L}(\lambda, x),
$$

where each $\Phi_{K, L}$, with $K+L<\ell \alpha+\beta-\mathbf{1}$, is independent of at least some coordinate $x_{j}$. Using this, and changing to polar coordinates, one readily checks that the first sum on the right hand side does not contribute to the integral (8) . Substituting the second sum into (8) the singularity of the integrand vanishes and one may put $\lambda=0$ and let $\epsilon \rightarrow 0^{+}$to obtain

$$
\begin{aligned}
& \lim _{\epsilon \rightarrow 0^{+}} \int_{r, \theta} \chi\left(r^{2 \alpha} / \epsilon\right) \sum_{K+L=\ell \alpha+\beta-1} e^{i \theta \cdot(K-L-\ell \alpha-\beta)} \Phi_{K, L}\left(0, r e^{i \theta}\right) d r d \theta \\
& \quad=\chi(\infty) \int_{r, \theta} \sum_{K+L=\ell \alpha+\beta-1} e^{i \theta \cdot(K-L-\ell \alpha-\beta)} \Phi_{K, L}\left(0, r e^{i \theta}\right) d r d \theta
\end{aligned}
$$

Computing $\left(1 /\left(f^{\ell} h\right)\right)[V] . \varphi$ in the same way, using the same desingularization and choice of coordinates one easily checks that this last integral is what one gets (in the $x$-chart).

Let $Q$ be a holomorphic differential operator in $X$ and put $\mu=$ $(1 / h)[V]$. It is clear that $\overline{\mathcal{J}}_{V} \cdot Q(\mu)=0$ and that $\operatorname{Supp}(\bar{\partial} Q(\mu)) \subseteq h^{-1}(0)$. Moreover, from the lemma it follows that $Q(\mu)$ has the Standard Extension Property. In fact, let $\{f=0\}$ be a hypersurface in $X$ such that $V \backslash\{f=0\}$ is dense in $V$, let $\chi$ be a smooth regularization of the characteristic function of $[1, \infty)$, and put $\chi^{\epsilon}=\chi\left(|f|^{2} / \epsilon\right)$. Then a simple computation shows that

$$
\chi^{\epsilon} \cdot Q(\mu)=Q\left(\chi^{\epsilon} \cdot \mu\right)+\sum_{j} Q_{j}\left(\frac{\chi_{j}^{\epsilon}}{f^{k_{j}}} \cdot \mu\right),
$$

where $Q_{j}$ are certain differential operators and $\chi_{j}^{\epsilon}=\chi_{j}\left(|f|^{2} / \epsilon\right)$ with $\chi_{j}$ smooth on $[0, \infty]$ and $\chi_{j}(\infty)=0$; cf. (6) . From the lemma it then follows that $\chi^{\epsilon} \cdot Q(\mu) \rightarrow Q(\mu)$.

With these facts in mind we define the Coleff-Herrera currents on $V, \mathbf{C H}_{V}$, and the Coleff-Herrera currents on $V$ with pole along $S$, $\mathbf{C H}_{V}[* S]$, for hypersurfaces $S \subseteq X$ such that $V \backslash S$ is dense in $V$. 
Definition 3. (The sheaves $\mathbf{C H}_{V}$ and $\mathbf{C H}_{V}[* S]$.)

A current $\mu$ of bidegree $(0, P)$ on an open set $U \subseteq X$ is a section of $\mathbf{C H}_{V}$ over $U$ if

1) $\mu$ has the Standard Extension Property,

2) $\overline{\mathcal{J}}_{V} \cdot \mu=0$,

3) $\bar{\partial} \mu=0$.

If $\mu$ satisfies 1$), 2$ ), and $\operatorname{Supp}(\bar{\partial} \mu) \subseteq S$, then we say that $\mu$ is a section of $\mathbf{C H}_{V}[* S]$ over $U$.

We have the following local representation of Dolbeault-Lelong type of currents in $\mathbf{C H}_{V}$, and consequently of currents in $\mathbf{C H}_{V}[* S]$; see below. The slick proof is taken from [2].

Proposition 4. Let $X$ be a neighborhood of the closure of the unit ball $\mathbb{B} \subset \mathbb{C}^{N}$ and let $\mu \in \mathbf{C H}_{V}$. In $\mathbb{B}$, there is a holomorphic differential operator $Q$, a holomorphic $n$-form $\vartheta$, and a holomorphic function $h$ with $V$-polar zero set, such that

$$
\mu \cdot(\varphi \wedge d z)=\lim _{\epsilon \rightarrow 0^{+}} \int_{V \cap\{|h|>\epsilon\}} \frac{Q(\varphi) \wedge \vartheta}{h}, \quad \varphi \in \mathscr{D}_{0, n}(\mathbb{B}) .
$$

Proof. Let $y \in V$ and assume that we have local coordinates $w=$ $\left(w^{\prime} ; w^{\prime \prime}\right)=\left(w_{1}, \ldots, w_{P} ; w_{P+1}, \ldots, w_{N}\right)$ so that $V=\left\{w^{\prime}=0\right\}$ close to $y$. If $1 \leq j \leq P$ we have by 2 ) in Definition 3 that $\bar{w}_{j} \mu=0$, and so, by 3 ), we get $d \bar{w}_{j} \wedge \mu=\bar{\partial}\left(\bar{w}_{j} \mu\right)=0$. It follows, for any function $\phi$ with support close to $y$, that $\mu \cdot\left(\phi d \bar{w}_{I} \wedge d w\right)=0$ if $d \bar{w}_{I} \neq \pm d \bar{w}^{\prime \prime}$. Let $\Pi: \mathbb{C}_{w}^{N} \rightarrow \mathbb{C}_{w^{\prime \prime}}^{n}$ be the standard projection and define

$$
a_{\alpha}\left(w^{\prime \prime}\right)=\Pi_{*}\left(w^{\alpha} d w^{\prime} \wedge \mu / \alpha !\right)
$$

for $\alpha=\left(\alpha^{\prime}, 0\right)$. Since $\mu$ is $\bar{\partial}$-closed, the $a_{\alpha}$ must be holomorphic. We claim that

$$
\mu=\frac{1}{(2 \pi i)^{P}} \sum_{\alpha=\left(\alpha^{\prime}, 0\right)} a_{\alpha}\left(w^{\prime \prime}\right) \bar{\partial} \frac{1}{w_{P}^{\alpha_{P}+1}} \wedge \cdots \wedge \bar{\partial} \frac{1}{w_{1}^{\alpha_{1}+1}}
$$

close to $y$, where the sum ranges over $\alpha$ with $|\alpha|$ less than or equal to the order, $M$, of $\mu$ on $\overline{\mathbb{B}}$. Given the claim, the proposition easily follows for test forms with support close to $y$. In fact, by the Poincarè-Lelong formula, we may take $(-1)^{n P} \sum_{\alpha=\left(\alpha^{\prime}, 0\right)} a_{\alpha}\left(w^{\prime \prime}\right) \partial^{\alpha}$ as the differential operator, let $\vartheta=d w^{\prime \prime}$, and $h=1$. To prove the claim, we note that it suffices to check it for test forms $\phi d \bar{w}^{\prime \prime} \wedge d w$ by the observation in the beginning of the proof. We write $\phi$ as a Taylor sum

$$
\phi=\sum_{\alpha=\left(\alpha^{\prime}, 0\right)} \frac{\partial^{|\alpha|} \phi}{\partial w^{\alpha}}\left(0, w^{\prime \prime}\right) \frac{w^{\alpha}}{\alpha !}+\overline{\mathcal{J}}_{V}+\mathcal{O}\left(\left|w^{\prime}\right|^{M+1}\right) .
$$


Noting that $\left|w^{\prime}\right|^{M+1} \mu=0$ and that $\overline{\mathcal{J}}_{V} \cdot \mu=0$, by 2) in Definition 3. the claim now follows from the definition of the $a_{\alpha}$ and a simple computation.

To obtain global $Q, \vartheta$, and $h$ we proceed as follows. We choose $H_{1}, \ldots, H_{P} \in \mathcal{J}_{V}(\mathbb{B})$ and coordinates $z=\left(z^{\prime}, z^{\prime \prime}\right)$ for $\mathbb{B}$ such that $\tilde{h}:=$ $\operatorname{det}\left(\partial H / \partial z^{\prime}\right)$ is generically non-vanishing on every component of $V$, i.e., $\tilde{h}^{-1}(0)$ is $V$-polar. Outside $\{\tilde{h}=0\}$ we can then make the change of variables $w=\left(w^{\prime}, w^{\prime \prime}\right):=\left(H, z^{\prime \prime}\right)$. Note however that $w_{j} \in \mathcal{O}(\mathbb{B})$ for all $j$. Outside $\{\tilde{h}=0\}$ we thus have a realization (11) of $\mu$ with $a_{\alpha} \in \mathcal{O}(\mathbb{B})$. Moreover, since $\partial / \partial w^{\prime}={ }^{t}\left(d H / d z^{\prime}\right)^{-1} \partial / \partial z^{\prime}$, we see that

$$
Q^{\prime}:=(-1)^{n P} \tilde{h}^{k} \sum_{\alpha=\left(\alpha^{\prime}, 0\right)} a_{\alpha}\left(w^{\prime \prime}\right) \partial_{w^{\prime}}^{\alpha}
$$

is a holomorphic differential operator in $\mathbb{B}$ if $k$ is large enough; recall that $|\alpha| \leq M$. For large enough $\ell$ we then define the holomorphic differential operator $Q$ by $Q(\phi)=\tilde{h}^{\ell} Q^{\prime}(\phi / \tilde{h})$. Letting $h=\tilde{h}^{k+\ell}$ and $\vartheta=d z^{\prime \prime}$, the formula (10) then follows from (11) if $\varphi$ has support outside $\{h=0\}$. But both $\mu$ and the current defined by the right hand side of (10) has the Standard Extension Property, by 1) in Definition 3 and the comment after the proof of Lemma 2 respectively, and so the proposition follows.

This proposition makes it possible to divide Coleff-Herrera currents by holomorphic functions. Let $\mu \in \mathbf{C H}_{V}$ and let $f$ be a holomorphic function such that $V \backslash f^{-1}(0)$ is dense in $V$. Given a local representation (10) of $\mu$ we put

$$
\frac{1}{f} \mu \cdot(\varphi \wedge d z)=\lim _{\epsilon \rightarrow 0^{+}} \int_{V \cap\{|h f|>\epsilon\}} \frac{Q(\varphi / f) \wedge \vartheta}{h} .
$$

It is clear that $(1 / f) \mu \in \mathbf{C H}_{V}\left[* f^{-1}(0)\right]$. On the other hand, if $\gamma \in$ $\mathbf{C H}_{V}\left[* f^{-1}(0)\right]$, then (at least locally) for some large $k$ we have $\tau=$ $f^{k} \gamma \in \mathbf{C H}_{V}$. Thus, $\gamma=\left(1 / f^{k}\right) \tau$ for some $\tau \in \mathbf{C H}_{V}$. It follows that we have representations (10) also for currents $\gamma \in \mathbf{C H}_{V}[* S]$ if $V \backslash S$ is dense in $V$ and that $(1 / f) \gamma$ is defined. From Lemma 2 and the technique of its proof it follows that

$$
\frac{1}{f} \mu=\lim _{\epsilon \rightarrow 0^{+}} \frac{\chi\left(|f|^{2} / \epsilon\right)}{f} \mu=\left.\frac{|f|^{2 \lambda}}{f} \mu\right|_{\lambda=0}, \quad \mu \in \mathbf{C H}_{V}[* S]
$$

cf. also (9).

Proposition 5. Let $S \subseteq X$ be a hypersurface such that $V \backslash S$ is dense in $V$. Then

$$
\bar{\partial}: \mathbf{C H}_{V}[* S] \rightarrow \mathbf{C H}_{V \cap S}
$$


Remark 6. This mapping actually fits into a long exact sequence, see, e.g., [12]. In particular, if $S$ is $V$-polar, then

$$
0 \rightarrow \mathbf{C H}_{V} \hookrightarrow \mathbf{C H}_{V}[* S] \stackrel{\bar{\partial}}{\longrightarrow} \mathbf{C H}_{V \cap S} \longrightarrow \mathcal{H}_{[V]}^{P+1}\left(\mathcal{O}_{X}\right) \rightarrow 0
$$

is exact. Here, $\mathcal{H}_{[V]}^{P+1}\left(\mathcal{O}_{X}\right)$ is (isomorphic to) the cohomology group $\operatorname{Ker}_{\bar{\partial}}\left(\mathcal{C}_{V}^{0, P+1}\right) / \bar{\partial}\left(\mathcal{C}_{V}^{0, P}\right)$, where $\mathcal{C}_{V}^{0, *}$ are the currents on $X$ of bidegree $(0, *)$ with support contained in $V$. If, in addition, $V$ is Cohen-Macaulay then this group vanishes and the mapping of Proposition 5 becomes surjective.

Proof. We will start by indicating how to prove the following

Claim: Let $f, g, h$ be holomorphic functions such that $h^{-1}(0)$ is $V$ polar and $V \backslash f^{-1}(0)$ is dense in $V$. Then

(14) $\lambda \mapsto \frac{\bar{\partial}|f|^{2 \lambda}}{f^{\ell}} \wedge \frac{1}{h}[V]$ has an analytic continuation as a current,

$$
\left.\frac{\chi\left(|g|^{2} / \epsilon\right)}{g^{k}} \frac{\bar{\partial}|f|^{2 \lambda}}{f^{\ell}} \wedge \frac{1}{h}[V]\right|_{\lambda=0} \rightarrow 0, \quad \text { as } \quad \epsilon \rightarrow 0^{+}
$$

if $\chi \in C^{\infty}([0, \infty])$ and vanishes both close to 0 and $\infty$. Moreover, if $(f, g)$ defines a complete intersection on $V$ and $\chi$ is a smooth regularization of the characteristic function of $[1, \infty)$ then

$$
\left.\left.\chi\left(|g|^{2} / \epsilon\right) \frac{\bar{\partial}|f|^{2 \lambda}}{f^{\ell}} \wedge \frac{1}{h}[V]\right|_{\lambda=0} \rightarrow \frac{\bar{\partial}|f|^{2 \lambda}}{f^{\ell}} \wedge \frac{1}{h}[V]\right|_{\lambda=0},
$$

as $\epsilon \rightarrow 0^{+}$. If instead $g \in \mathcal{J}_{V \cap f^{-1}(0)}$, then

$$
\left.\bar{g} \frac{\bar{\partial}|f|^{2 \lambda}}{f^{\ell}} \wedge \frac{1}{h}[V]\right|_{\lambda=0} \rightarrow 0 .
$$

To prove (14), one computes in a resolution $\pi: \mathcal{V} \rightarrow V$ such that $\left\{\pi^{*} f \cdot \pi^{*} h=0\right\}$ has normal crossings in the manifold $\mathcal{V}$ and one chooses, preferably, local coordinates, $x$, such that $\pi^{*} h=x^{\beta}$ and $\pi^{*} f=u x^{\alpha}$, where $u$ is holomorphic and invertible. To prove (15) and (17) one proceeds similarly; one first computes $\left.\left(\bar{\partial}|f|^{2 \lambda} / f^{\ell}\right) \wedge(1 / h)[V]\right|_{\lambda=0}$ in a resolution $\pi: \mathcal{V} \rightarrow V$ such that $\left\{\pi^{*} f \cdot \pi^{*} h \cdot \pi^{*} g=0\right\}$ has normal crossings, preferably using coordinates such that $\pi^{*} f=u x^{\alpha}, \pi^{*} h=$ $v x^{\beta}$, and $\pi^{*} g=x^{\gamma}$. Then it is not too hard to verify (15) and (17). It is a bit more delicate to prove (16) since the assumption about complete intersection has to be used properly. Let $\varphi$ be a $(n, n-1)$-test form in the base space $X$. On a resolution manifold $\mathcal{V}$ as the one above, one chooses an atlas of local coordinates with the properties stated above, and moreover, so that $\pi^{*} \varphi=\phi d \bar{x}^{\prime} \wedge d x$, where $d \bar{x}^{\prime}=d \bar{x}_{2} \wedge \cdots \wedge d \bar{x}_{n}$. 
The trick is now to show that $\left.\left(\bar{\partial}|f|^{2 \lambda} / f^{\ell}\right) \wedge(1 / h)[V] \cdot \varphi\right|_{\lambda=0}$ equals

$$
\left.\left.\sum_{\substack{x_{1} \mid x^{\alpha} \\ x_{1} \nmid x^{\gamma}}} \int \frac{\bar{\partial}\left|x^{\alpha}\right|^{2 \lambda}\left|x^{\beta}\right|^{2 s}}{x^{\ell \alpha+\beta}} \wedge \frac{|u|^{2 \lambda}|v|^{2 s}}{u v} \rho_{x} \phi d \bar{x}^{\prime} \wedge d x\right|_{s=0}\right|_{\lambda=0}
$$

where $\left\{\rho_{x}\right\}$ is a partition of unity on $\mathcal{V}$. That is, that only charts on $\mathcal{V}$ such that $x_{1} \mid \pi^{*} f$ and $x_{1} \nmid \pi^{*} g$ contribute. That $x_{1}$ has to divide $\pi^{*} f$ is obvious. If, in addition, $x_{1}$ divides $\pi^{*} g$, then $\left\{x_{1}=0\right\} \subseteq$ $\pi^{-1}\{f=g=0\} \cap V$. Since $\{f=g=0\} \cap V$ has dimension $n-2$ it follows that any anti-holomorphic $n-1$-form in $X$ has a vanishing pullback to $\{f=g=0\} \cap V$. Thus, $\pi^{*} \varphi$ has a vanishing pullback to $\left\{x_{1}=0\right\}$. It follows that $\phi=\bar{x}_{1} \tilde{\phi}$ for some smooth $\tilde{\phi}$. Using this one easily shows that charts where $x_{1}$ divides both $\pi^{*} f$ and $\pi^{*} g$ do not contribute. With this in mind it is not very difficult to show that $\left.\chi\left(|g|^{2} / \epsilon\right)\left(\bar{\partial}|f|^{2 \lambda} / f^{\ell}\right) \wedge(1 / h)[V] \cdot \varphi\right|_{\lambda=0}$ tends to (18) as $\epsilon \rightarrow 0^{+}$, which then proves (16).

It is now easy to prove the proposition. Let $\mu \in \mathbf{C H}_{V}[* S]$; it is a local problem to show that $\bar{\partial} \mu \in \mathbf{C H}_{V \cap S}$. Choose a holomorphic function $f$ and a $\tau \in \mathbf{C H}_{V}$ such that $S=f^{-1}(0)$ and $\mu=(1 / f) \tau$. From (13) we have $\mu=\left.\left(|f|^{2 \lambda} / f\right) \tau\right|_{\lambda=0}$, and for $\mathfrak{R e} \lambda>>1$ we have $\bar{\partial}\left(\left(|f|^{2 \lambda} / f\right) \tau\right)=\left(\bar{\partial}|f|^{2 \lambda} / f\right) \tau$. From (14), the last expression also has an analytic continuation, and so $\bar{\partial} \mu=\left.\left(\bar{\partial}|f|^{2 \lambda} / f\right) \tau\right|_{\lambda=0}$. Using a representation (10) of $\tau$ and (14) and (17) one easily sees that $\bar{g} \bar{\partial} \mu=0$ if $g \in \mathcal{J}_{V \cap S}$ (recall that $S=f^{-1}(0)$ ). Similarly, if $\tau$ is represented by (10) it follows from (14), (15), and (16) that $\chi\left(|g|^{2} / \epsilon\right) \bar{\partial} \mu \rightarrow \bar{\partial} \mu$, as $\epsilon \rightarrow 0^{+}$; i.e., that $\bar{\partial} \mu$ has the Standard Extension Property.

Definition 7. Let $\mu \in \mathbf{C H}_{V}[* S]$ and let $f$ be a holomorphic function such that $V \backslash f^{-1}(0)$ is dense in $V$ and $V \cap S \backslash f^{-1}(0)$ is dense in $V \cap S$. We define $\bar{\partial}(1 / f) \wedge \mu$ by

$$
\bar{\partial} \frac{1}{f} \wedge \mu=\bar{\partial}\left(\frac{1}{f} \mu\right)-\frac{1}{f} \bar{\partial} \mu .
$$

That this definition makes sense follows from Proposition 5. It is intuitively clear that $\bar{\partial}(1 / f) \wedge \mu \in \mathbf{C H}_{V \cap f^{-1}(0)}[* S]$ but it is not immediate from the definition. However, letting $\mu=(1 / g) \tau$, where $\tau \in \mathbf{C H}_{V}$ and $g^{-1}(0)=S$, we get from Proposition 8 that

$$
\begin{aligned}
\left|\bar{\partial} \frac{\chi\left(|f|^{2} / \epsilon_{1}\right)}{f} \wedge \frac{\chi\left(|g|^{2} / \epsilon_{2}\right)}{g} \tau-\bar{\partial} \frac{1}{f} \wedge \frac{1}{g} \tau\right| & \\
\leq C \epsilon_{2}^{\omega}+\mid \bar{\partial} \frac{\chi\left(|f|^{2} / \epsilon_{1}\right)}{f} & \wedge \mu-\bar{\partial} \frac{1}{f} \wedge \mu \mid \\
& \leq C \epsilon_{2}^{\omega}+\left|\bar{\partial}\left(\frac{\chi\left(|f|^{2} / \epsilon_{1}\right)}{f} \wedge \mu\right)-\bar{\partial}\left(\frac{1}{f} \wedge \mu\right)\right|
\end{aligned}
$$




$$
+\left|\frac{\chi\left(|f|^{2} / \epsilon_{1}\right)}{f} \bar{\partial} \mu-\frac{1}{f} \bar{\partial} \mu\right| \lesssim \epsilon_{1}^{\omega}+\epsilon_{2}^{\omega},
$$

where $\chi$ is a smooth regularization of the characteristic function of $[1, \infty)$. Thus, $\left(\bar{\partial} \chi\left(|f|^{2} / \epsilon_{1}\right) / f\right) \wedge\left(\chi\left(|g|^{2} / \epsilon_{2}\right)\right) \tau$ converges unrestrictedly to $\bar{\partial}(1 / f) \wedge \mu$. First letting $\epsilon_{2} \rightarrow 0$ and then letting $\epsilon_{1} \rightarrow 0$ we then see that $\bar{\partial}(1 / f) \wedge \mu=(1 / g) \bar{\partial}((1 / f) \tau) \in \mathbf{C H}_{V \cap f^{-1}(0)}[* S]$ by Proposition 5 .

If $\mu \in \mathbf{C H}_{V}$ and $f=\left(f_{1}, \ldots, f_{q}\right): X \rightarrow \mathbb{C}^{q}$ is holomorphic such that $\left(f_{1}, \ldots, f_{p}, f_{j}\right)$ defines a complete intersection on $V$ for $p+1 \leq j \leq q$ we have thus given a meaning to

$$
\bar{\partial} \frac{1}{f_{1}} \wedge \cdots \wedge \bar{\partial} \frac{1}{f_{p}} \cdot \frac{1}{f_{p+1}} \cdots \frac{1}{f_{q}} \wedge \mu .
$$

It follows from Theorem 1 that this product, apart from being alternating in $f_{1}, \ldots, f_{p}$ as it should, is independent of the ordering of the tuple $f$ and, moreover, that it coincides with the definition of Coleff-Herrera and Passare.

\section{THE KEY PROPOSITION}

In this section we prove the key proposition needed to prove our main theorem. The proof of the proposition relies on a Whitney type division lemma for the pullback of anti-holomorphic forms through modifications. This lemma appear also in [28].

Throughout this section our considerations are local; $X=\mathbb{B}$ is the unit ball in $\mathbb{C}^{N}$ and $V$ is an analytic set of pure dimension $n$ (and codimension $P=N-n$ ) defined in a neighborhood of $\mathbb{B}$.

Proposition 8. Let $V \subseteq \mathbb{B}$ be an analytic set of pure dimension $n$, $S \subset \mathbb{B}$ a V-polar set, and $f=\left(f_{1}, \ldots, f_{q}\right): \mathbb{B} \rightarrow \mathbb{C}^{q}$ a holomorphic mapping such that $\left(f_{1}, \ldots, f_{p}, f_{j}\right)$ defines a complete intersection on $V$ for all $j=p+1, \ldots, q$. Let also $\chi_{j}, 1 \leq j \leq q$, be smooth on $[0, \infty]$ and vanish to order $\ell_{j}$ at 0 . Then for any $\mu \in \mathbf{C H}_{V}[* S]$ and $\varphi \in \mathscr{D}_{0, n-p}(\mathbb{B})$ we have

$$
\begin{array}{r}
\left|\frac{\bar{\partial} \chi_{1}^{\epsilon} \wedge \cdots \wedge \bar{\partial} \chi_{p}^{\epsilon} \chi_{p+1}^{\epsilon} \cdots \chi_{q-1}^{\epsilon}}{f_{1}^{\ell_{1}} \cdots f_{q-1}^{\ell_{q}-1}}\left(\frac{\chi_{q}^{\epsilon}}{f_{q}^{\ell_{q}}}-\frac{1}{f_{q}^{\ell_{q}}}\right) \wedge \mu .(\varphi \wedge d z)\right| \leq \\
\leq C\|\varphi\|_{M} \epsilon_{q}^{\omega},
\end{array}
$$

where $\chi_{j}^{\epsilon}=\chi_{j}\left(\left|f_{j}\right|^{2} / \epsilon_{j}\right), M$ and $\omega$ are positive constants that only depend on $f$ and $\operatorname{Supp}(\varphi)$, while the positive constant $C$ also might depend on the $C^{M}$-norm of the $\chi_{j}$-functions.

Proof. We fix a representation (10) of $\mu$ (or rather its "analytic continuation" counterpart) and write

$$
\mathcal{I}_{f, \mu}^{\varphi}(\epsilon):=\frac{\bar{\partial} \chi_{1}^{\epsilon} \wedge \cdots \wedge \bar{\partial} \chi_{p}^{\epsilon} \chi_{p+1}^{\epsilon} \cdots \chi_{q}^{\epsilon}}{f_{1}^{\ell_{1}} \cdots f_{q}^{\ell_{q}}} \wedge \mu \cdot(\varphi \wedge d z)
$$




$$
=\left.\int_{V} \frac{|h|^{2 \lambda}}{h} Q\left(\frac{\bar{\partial} \chi_{1}^{\epsilon} \wedge \cdots \wedge \bar{\partial} \chi_{p}^{\epsilon} \chi_{p+1}^{\epsilon} \cdots \chi_{q}^{\epsilon}}{f_{1}^{\ell_{1}} \cdots f_{q}^{\ell_{q}}} \wedge \varphi\right) \wedge \vartheta\right|_{\lambda=0} .
$$

Since expressions like $\chi^{\epsilon} / f^{\ell}$ essentially are invariant under holomorphic differential operators, cf. (6) , and since $\bar{\partial}$ commutes with such operators, the right hand side of (19) is, by Leibniz' rule, a finite sum of integrals of the same kind but with the holomorphic differential operator $Q$ omitted. We can therefore ignore $Q$ in the computations below.

By Hironaka's theorem, e.g., formulated as in [18] and [8], one can find, first an $n$-dimensional complex manifold $\tilde{V}$ and a proper holomorphic map $\pi_{1}: \tilde{V} \rightarrow V$ that defines a biholomorphism outside $V_{\text {sing }}$, and then (at least locally on $\tilde{V}$ ) a further $n$-dimensional complex manifold $\mathcal{V}$ and a proper holomorphic map $\pi_{2}: \mathcal{V} \rightarrow \tilde{V}$ such that $\mathcal{Z}:=$ $\pi_{2}^{-1}\left(\pi_{1}^{*} h \cdot \pi_{1}^{*} f_{1} \cdots \pi_{1}^{*} f_{q}=0\right)$ has normal crossings and $\pi_{2}$ is a biholomorphism outside $\mathcal{Z}$. Put $\pi=\pi_{1} \circ \pi_{2}$ and denote the pull-back under $\pi$ by $\hat{\imath}$, e.g., $\hat{h}=\pi^{*} h$. We choose a (sufficiently fine) finite partition of unity $\left\{\rho_{j}\right\}$ on $\operatorname{Supp}(\hat{\varphi})$ and local charts on the $\operatorname{Supp}\left(\rho_{j}\right)$ such that $\hat{h}, \hat{f}_{1}, \ldots, \hat{f}_{q}$ are monomials times invertible holomorphic functions. The right hand side of (19), (recall that we may ignore $Q$ ), is therefore equal to

$$
\left.\sum_{i} \int_{\mathcal{V}} \frac{|\hat{h}|^{2 \lambda}}{\hat{h}} \frac{\bar{\partial} \chi_{1}^{\epsilon} \wedge \cdots \wedge \bar{\partial} \chi_{p}^{\epsilon} \chi_{p+1}^{\epsilon} \cdots \chi_{q}^{\epsilon}}{\hat{f}_{1}^{\ell_{1}} \cdots \hat{f_{q}^{\ell_{q}}}} \wedge \hat{\varphi} \wedge \hat{\vartheta} \rho_{i}\right|_{\lambda=0}
$$

Moreover, we may assume that $\varphi$ is of the form $\varphi_{I} d \bar{z}_{I},|I|=n-$ $p$, and so we can write $\hat{\varphi}=\eta \cdot \phi_{1}$, where $\eta=\hat{\varphi}_{I} \in \mathscr{D}_{0,0}(\mathcal{V})$ and $\phi_{1}=\widehat{d \bar{z}}_{I}$ is an anti-holomorphic $n-p$-form on $\mathcal{V}$. We now consider one term of (20), we drop the subscript $i$ from $\rho_{i}$, and we put $\phi_{2}:=$ $\eta \hat{\vartheta} \rho$. In a neighborhood of $\operatorname{Supp}(\rho)$ we have local coordinates $x$ such that $\hat{f}_{j}=u_{j} x^{\alpha(j)}$, where $u_{j}$ are invertible and holomorphic. We let $m$ be the number of vectors in a maximal linearly independent subset of $\{\alpha(1), \ldots, \alpha(p)\}$, and we assume for notational convenience that $\alpha(1), \ldots, \alpha(m)$ are linearly independent. As in [22], p. 46, we can define new coordinates, still denoted $x$, so that $u_{1}=\cdots=u_{m}=1$. For $m+1 \leq j \leq p$ we write $\bar{\partial} \chi_{j}^{\epsilon}=\tilde{\chi}_{j}^{\epsilon} \cdot\left(d \bar{x}^{\alpha(j)} / \bar{x}^{\alpha(j)}+d \bar{u}_{j} / \bar{u}_{j}\right)$, where $\tilde{\chi}_{j}(t)=t \chi_{j}^{\prime}(t)$ are smooth on $[0, \infty]$, vanish to order $\ell_{j}$ at 0 , and map $\infty$ to 0 . We will omit the tildes in the computations below, and hence, with abuse of notation, the term of (20) under consideration can be written

$$
\left.\int_{\mathcal{V}} \frac{|\hat{h}|^{2 \lambda}}{\hat{h}} \frac{\bar{\partial} \chi_{1}^{\epsilon} \wedge \cdots \wedge \bar{\partial} \chi_{m}^{\epsilon} \chi_{m+1}^{\epsilon} \cdots \chi_{q}^{\epsilon}}{\hat{f}_{1}^{\ell_{1}} \cdots \hat{f}_{q}^{\ell_{q}}} \bigwedge_{m+1}^{p}\left(\frac{d \bar{x}^{\alpha(j)}}{\bar{x}^{\alpha(j)}}+\frac{d \bar{u}_{j}}{\bar{u}_{j}}\right) \phi_{1} \wedge \phi_{2}\right|_{\lambda=0}
$$

From exterior algebra it follows that $d \bar{x}^{\alpha(1)} \wedge \cdots \wedge d \bar{x}^{\alpha(m)} \wedge d \bar{x}^{\alpha(j)}=0$ if $m+1 \leq j \leq p$ since $\alpha(1), \ldots, \alpha(m), \alpha(j)$ are linearly dependent. 
Thus, since $\bar{\partial} \chi_{1}^{\epsilon} \wedge \cdots \wedge \bar{\partial} \chi_{m}^{\epsilon}$ is proportional to $d \bar{x}^{\alpha(1)} \wedge \cdots \wedge d \bar{x}^{\alpha(m)}$, we may erase the factors $d \bar{x}^{\alpha(j)} / \bar{x}^{\alpha(j)}, m+1 \leq j \leq p$ from (21). We now let $\mathcal{K}$ be the set of indices $i$ such that $x_{i}$ divides some $x^{\alpha(j)}$ with $p+1 \leq j \leq q$ and we apply Lemma 9 with in data $\mathcal{K}$ and $d \bar{z}_{I}$. We find that we may replace $d \bar{u}_{m+1} \wedge \cdots \wedge d \bar{u}_{p} \wedge \phi_{1}$ by an anti-holomorphic form $\xi=\sum_{|J|=n-m} \xi_{J} d \bar{x}_{J}$, which has the property that each $\xi_{J}$ is divisible by all $\bar{x}_{i}, i \in \mathcal{K}$, without affecting the integral (21). We may of course assume that $\xi$ consists of one term only, and for notational convenience we assume that $\xi=\xi^{\prime} d \bar{x}_{m+1} \wedge \cdots \wedge d \bar{x}_{n}$. We assume, also for simplicity, that $\mathcal{K} \backslash\{m+1, \ldots, n\}=\{k+1, \ldots, m\}$ so that $\xi^{\prime}$ may be written $\bar{x}_{k+1} \cdots \bar{x}_{m} \xi^{\prime \prime}=: \bar{x}^{\mathbf{1}_{k}^{m}} \xi^{\prime \prime}$ for some anti-holomorphic function $\xi^{\prime \prime}$. We can now re-write the integral (21) as

$$
\left.\int_{\mathcal{V}} \frac{|\hat{h}|^{2 \lambda}}{\hat{h}} \frac{\bar{\partial} \chi_{1}^{\epsilon} \wedge \cdots \wedge \bar{\partial} \chi_{m}^{\epsilon} \chi_{m+1}^{\epsilon} \cdots \chi_{q}^{\epsilon}}{\hat{f}_{1}^{\ell_{1}} \cdots \hat{f}_{q}^{\ell_{q}}} \frac{\bar{x}_{k}^{m} \xi^{\prime \prime} \bigwedge_{m+1}^{n} d \bar{x}_{j} \wedge \phi_{2}}{\bar{u}_{m+1} \cdots \bar{u}_{p}}\right|_{\lambda=0}
$$

Now consider the form $\bar{\partial} \chi_{1}^{\epsilon} \wedge \cdots \wedge \bar{\partial} \chi_{m}^{\epsilon} \wedge_{m+1}^{n} d \bar{x}_{j}$. We write $\bar{\partial}=\bar{\partial}^{\prime}+\bar{\partial}^{\prime \prime}$, where $\bar{\partial}^{\prime}$ differentiates with respect to the variables $x^{\prime}=\left(x_{1}, \ldots, x_{k}\right)$ and $\bar{\partial}^{\prime \prime}$ differentiates with respect to the variables $x^{\prime \prime}=\left(x_{k+1}, \ldots, x_{n}\right)$, and we compute:

$$
\begin{aligned}
& \bar{\partial} \chi_{1}^{\epsilon} \wedge \cdots \wedge \bar{\partial} \chi_{m}^{\epsilon} \bigwedge_{m+1}^{n} d \bar{x}_{j}=\left(\bar{\partial}^{\prime} \chi_{1}^{\epsilon}+\bar{\partial}^{\prime \prime} \chi_{1}^{\epsilon}\right) \wedge \cdots \wedge\left(\bar{\partial}^{\prime} \chi_{m}^{\epsilon}+\bar{\partial}^{\prime \prime} \chi_{m}^{\epsilon}\right) \bigwedge_{m+1}^{n} d \bar{x}_{j} \\
& =\sum_{\substack{i_{1}<\cdots<i_{k} \\
i_{k+1}<\cdots<i_{m}}} \operatorname{sign}\left(j \mapsto i_{j}\right) \bar{\partial}^{\prime} \chi_{i_{1}}^{\epsilon} \wedge \cdots \wedge \bar{\partial}^{\prime} \chi_{i_{k}}^{\epsilon} \wedge \bar{\partial}^{\prime \prime} \chi_{i_{k+1}}^{\epsilon} \wedge \cdots \wedge \bar{\partial}^{\prime \prime} \chi_{i_{m}}^{\epsilon} \bigwedge_{m+1}^{n} d \bar{x}_{j}
\end{aligned}
$$

Let us consider the first term in this sum. It equals

$$
\operatorname{det} \tilde{A} \bar{\partial} \chi_{1}^{\epsilon} \wedge \cdots \wedge \bar{\partial} \chi_{k}^{\epsilon} \wedge \frac{d \bar{x}_{k+1} \wedge \cdots \wedge d \bar{x}_{m}}{\bar{x}_{k+1} \cdots \bar{x}_{m}} \tilde{\chi}_{k+1}^{\epsilon} \cdots \tilde{\chi}_{m}^{\epsilon} \bigwedge_{m+1}^{n} d \bar{x}_{j},
$$

where $\tilde{A}$ is the $(m-k) \times(m-k)$-matrix $\left(\alpha(i)_{j}\right)_{i, j=k+1}^{m}$ and $\tilde{\chi}_{j}^{\epsilon}=$ $\left(\left|\hat{f}_{j}\right|^{2} / \epsilon_{j}\right) \cdot \chi_{j}^{\prime}\left(\left|\hat{f}_{j}\right|^{2} / \epsilon_{j}\right)$. (As usual, we omit the tildes below.) The other terms in the sum on the right hand side of (23) are of the same type. In particular, each such term has $\bar{x}_{k+1} \cdots \bar{x}_{m}=\bar{x}_{k}^{m}$ as denominator. Recall also that $\hat{h}=v x^{\beta}$ with $v$ invertible and holomorphic. Substituting (23) into (22) we thus obtain finitely many integrals of the type

$$
\left.\int_{\mathcal{V}} \frac{\bar{\partial} \chi_{1}^{\epsilon} \wedge \cdots \wedge \bar{\partial} \chi_{k}^{\epsilon}}{x^{\ell \alpha+\beta}}\left|v x^{\beta}\right|^{2 \lambda} \chi_{k+1}^{\epsilon} \cdots \chi_{q}^{\epsilon} \bigwedge_{k+1}^{n} d \bar{x}_{j} \wedge \psi d x\right|_{\lambda=0}
$$


where $\psi d x=\xi^{\prime \prime} \phi_{2} /\left(v u_{m+1}^{\ell_{m+1}} \cdots u_{q}^{\ell_{q}} \bar{u}_{m+1} \cdots \bar{u}_{p}\right)$ and $\ell \alpha=\sum_{1}^{q} \ell_{j} \alpha(j)$. Note that $\psi$ has compact support, and, perhaps after scaling, we may assume it has support in the unit polydisc $\Delta$.

We now introduce the smoothing parameters

$$
t_{j}=\left|x^{\alpha(j)}\right|^{2} / \epsilon_{j}, \quad \text { for } \quad j=k+1, \ldots, q,
$$

and we put

$$
\Psi\left(\lambda, x, t_{k+1}, \ldots, t_{q}\right)=|v|^{2 \lambda} \Pi_{k+1}^{m} \chi_{i}\left(t_{i}\right) \cdot \Pi_{m+1}^{q} \chi_{j}\left(t_{j}\left|u_{j}\right|^{2}\right) \cdot \psi(x) .
$$

The function $\Psi(\lambda, x, t)$ is smooth on $\mathbb{C} \times \mathbb{C}^{n} \times[0, \infty]^{q-k}$ and by Lemma 6 in [27] it has the Taylor-like expansion

$$
\begin{aligned}
\Psi(\lambda, x, t)= & \sum_{K+L<\ell \alpha+\beta-1} x^{K} \bar{x}^{L} \Psi_{K, L}(\lambda, x, t) \\
& +\sum_{K+L=\ell \alpha+\beta-1} x^{K} \bar{x}^{L} \Psi_{K, L}(\lambda, x, t) .
\end{aligned}
$$

When doing this expansion we consider $t=\left(t_{k+1}, \ldots, t_{q}\right)$ as independent real variables and $\lambda$ as a parameter. If $K+L<\ell \alpha+\beta-\mathbf{1}$, the function $\Psi_{K, L}(\lambda, x, t)$ is independent of at least some coordinate $x_{j}$ and, moreover, we have the following explicit expression for the "remainder" part of the expansion:

$$
\begin{aligned}
& \sum_{K+L=\ell \alpha+\beta-1} x^{K} \bar{x}^{L} \Psi_{K, L}(\lambda, x, t) \\
= & \int_{y \in[0,1]^{n}} \frac{(\mathbf{1}-y)^{\ell \alpha+\beta-2}}{(\ell \alpha+\beta-\mathbf{2}) !} \frac{\partial^{|\ell \alpha+\beta-1|}}{\partial y^{\ell \alpha+\beta-1}} \Psi\left(\lambda, y_{1} x_{1}, \ldots, y_{n} x_{n}, t\right) d y .
\end{aligned}
$$

If we evaluate the smoothing parameters, i.e., let $t_{j}=\left|x^{\alpha(j)}\right|^{2} / \epsilon_{j}$, we have that $|v|^{2 \lambda} \chi_{k+1}^{\epsilon} \cdots \chi_{q}^{\epsilon} \psi=\Psi(\lambda, x, t)$ and we can substitute the decomposition (25) of $\Psi$ into (24). By changing to polar coordinates and using that $\Psi_{K, L}(\lambda, x, t)$ is independent of some $x_{j}$ if $K+L<\ell \alpha+\beta-\mathbf{1}$ it is not very hard to see that the first part of the expansion (25) does not contribute; see [22] p. 47,48 , for details. In polar coordinates, the integral (24) thus equals

$$
\left.\int_{r \in[0,1]^{n}} \mathscr{J}(\lambda, r, t) r^{2 \lambda \beta} d \chi_{1}^{\epsilon} \wedge \cdots \wedge d \chi_{k}^{\epsilon} \wedge d r_{k+1} \wedge \cdots \wedge d r_{n}\right|_{\lambda=0},
$$

where

$$
\mathscr{J}(\lambda, r, t)=c_{n, k} \int_{\theta \in[0,2 \pi)^{n}} \sum_{\substack{K+L=\\ \ell \alpha+\beta-1}} \Psi_{K, L}(\lambda, r, \theta, t) e^{i \theta \cdot\left(K-L-\ell \alpha-\beta+\mathbf{1}_{1}^{k}\right)} d \theta .
$$

Here, $\chi_{j}^{\epsilon}=\chi_{j}\left(r^{2 \alpha(j)} / \epsilon_{j}\right)$ for $j=1, \ldots, k, t_{j}=r^{2 \alpha(j)} / \epsilon_{j}$ for $j=k+$ $1, \ldots, q$, and $c_{n, k}=(-1)^{n^{2}} i^{n} 2^{n-k}$. Since the singularity has disappeared it is innocuous to put $\lambda=0$ in (27) and from now on we omit all 
occurrences of $\lambda$. We here also note the following properties of the function $\mathscr{J}(r, t)=\mathscr{J}(0, r, t)$.

a) $\mathscr{J}(r, t)$ is bounded on $[0,1]^{n} \times[0, \infty]^{q-k}$.

b) If some $t_{j}<\delta$ for some $k+1 \leq j \leq q$ then $|\mathscr{J}(r, t)| \leq C \delta$.

c) If $t_{j}>1 / \delta$ for some $k+1 \leq j \leq p$ then $|\mathscr{J}(r, t)| \leq C \delta$.

d)

$$
\begin{aligned}
\mid \mathscr{J}\left(r, t_{k+1}, \ldots, t_{q-1}, t_{q}\right)- & \mathscr{J}\left(r, t_{k+1}, \ldots, t_{q-1}, \infty\right) \mid \\
& \leq \begin{cases}C\|\psi\|_{|\ell \alpha+\beta-\mathbf{1}|} / t_{q}, & t_{q} \geq 1 \\
C\|\psi\|_{\mid \ell \alpha+\beta-1} \mid & t_{q} \leq 1\end{cases}
\end{aligned}
$$

Property a) follows easily from formula (26) and the definition of $\Psi(x, t)=$ $\Psi(0, x, t)$. Properties b) and c) follow by Taylor expanding $t_{j} \mapsto$ $\chi_{j}\left(t_{j}\left|u_{j}\right|^{2}\right)$ at 0 and $\infty$ respectively in the definition of $\Psi(x, t)$. Property d) follows by Taylor expanding $t_{q} \mapsto \chi_{j}\left(t_{q}\left|u_{q}\right|^{2}\right)$ at $\infty$ in the definition of $\Psi(x, t)$ and inspection in the formula (26) . We note also that the constant(s), $C$, depend on the $\left.C^{|\ell \alpha+\beta-1|}\right|_{\text {-norm of }} \chi_{j}$ if $u_{j} \neq 1$. Now, by Fubini's theorem we may write (27) as

$$
\int_{r^{\prime \prime} \in[0,1]^{n-k}}\left(\int_{r^{\prime} \in[0,1]^{k}} \mathscr{J}\left(r^{\prime}, r^{\prime \prime}, t\right) d \chi_{1}^{\epsilon} \wedge \cdots \wedge d \chi_{k}^{\epsilon}\right) d r_{k+1} \wedge \cdots \wedge d r_{n}
$$

and by property a), the modulus of the inner integral can be estimated by a constant times $\int_{s \in[0, \infty)^{k}}\left|d \chi_{1}\left(s_{1}\right) \wedge \cdots \wedge d \chi_{k}\left(s_{k}\right)\right| \leq C<\infty$ uniformly in all parameters. Thus, by Dominated Convergence, the study of possible limits of (27), and hence of (19), is reduced to the study of possible limits of the inner integral in (28) for fixed $r^{\prime \prime}=$ $\left(r_{k+1}, \ldots, r_{n}\right) \in[0,1]^{n-k}$. We note here also that $d \chi_{1}^{\epsilon} \wedge \cdots \wedge d \chi_{k}^{\epsilon}=$ $\operatorname{det} A \cdot \tilde{\chi}_{1}^{\epsilon} \cdots \tilde{\chi}_{k}^{\epsilon} d r_{1} \wedge \cdots \wedge d r_{k} /\left(r_{1} \cdots r_{k}\right)$, where $A$ is the $k \times k$-matrix $\left(2 \alpha(i)_{j}\right)_{i, j=1}^{k}$. We may thus assume that $A$ is invertible.

We want to estimate the difference $\left|\mathcal{I}_{f, \mu}^{\varphi}(\epsilon)-\lim _{\epsilon_{q} \rightarrow 0} \mathcal{I}_{f, \mu}^{\varphi}(\epsilon)\right|$ and by our computations this far it can be estimated by a finite sum of terms like

$$
\begin{aligned}
& \int_{[0,1]^{n-k}}\left(\int_{[0,1]^{k}}\left|\mathscr{J}\left(r^{\prime}, r^{\prime \prime}, t\right)-\lim _{\epsilon_{q} \rightarrow 0} \mathscr{J}\left(r^{\prime}, r^{\prime \prime}, t\right)\right| \cdot\left|d \chi_{1}^{\epsilon} \wedge \cdots \wedge d \chi_{k}^{\epsilon}\right|\right) d r^{\prime \prime} \\
& =\int_{[0,1]^{n-k}}\left(\int_{[0,1]^{k}}\left|\mathscr{J}\left(r^{\prime}, r^{\prime \prime}, t\right)-\mathscr{J}\left(r^{\prime}, r^{\prime \prime}, t\right)\right|_{t_{q}=\infty}|\cdot| d \chi_{1}^{\epsilon} \wedge \cdots \wedge d \chi_{k}^{\epsilon} \mid\right) d r^{\prime \prime} .
\end{aligned}
$$

The equality $\lim _{\epsilon_{q} \rightarrow 0} \mathscr{J}\left(r^{\prime}, r^{\prime \prime}, t\right)=\left.\mathscr{J}\left(r^{\prime}, r^{\prime \prime}, t\right)\right|_{t_{q}=\infty}$ holds for each fixed $r^{\prime \prime} \in(0,1]^{n-k}$ because $t_{q}=r^{2 \alpha(q)} / \epsilon_{q}$ and, since $\mathcal{K} \subseteq\{k+1, \ldots, n\}$, we have $\alpha(q)_{1}=\cdots=\alpha(q)_{k}=0$. By property $\left.\mathrm{d}\right)$ we can therefore estimate $\left|I_{f, \mu}^{\varphi}(\epsilon)-\lim _{\epsilon_{q} \rightarrow 0} I_{f, \mu}^{\varphi}(\epsilon)\right|$ by a finite sum of terms of the form

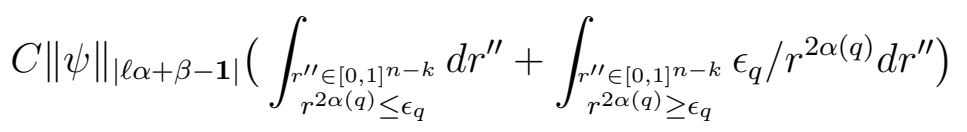




$$
\leq C\|\psi\|_{|\ell \alpha+\beta-1|} \epsilon_{q}^{\omega} .
$$

The last estimate follows from Lemmas 9 and 10 in [27, from which we also see that any $\omega<1 /(2|\alpha(q)|)$ works. To conclude the proof we just have to note that $\psi$ depends continuously on $\varphi$ in $C^{m}$-norm if $m$ is sufficiently large.

4.1. The division lemma. We keep the notation from the proof of Proposition 8 so that $\mathcal{V} \rightarrow V \subseteq \mathbb{B}$ is a modification, the pull-back under this map is denoted by $\hat{\imath}$, and $x$ are local coordinates on $\mathcal{V}$ such that $\hat{f}_{j}=x^{\alpha(j)}, 1 \leq j \leq m$, and $\hat{f}_{j}=u_{j} x^{\alpha(j)}, m+1 \leq j \leq q$. We recall also that our set-up in the proof of Proposition 8 implies that the exterior product of $\wedge_{1}^{m} d x^{\alpha(i)}$ with any $d x^{\alpha(j)}, m+1 \leq j \leq p$, is zero.

Lemma 9. Let $\mathcal{K} \subseteq\{1, \ldots, n\}$ be any set of indices $i$ such that $x_{i}$ divides some $x^{\alpha(j)}$ with $p+1 \leq j \leq q$. If $\sigma$ is an anti-holomorphic $n-p$ form in $\mathbb{B}$, then one can find, in the $x$-chart on $\mathcal{V}$, an anti-holomorphic $n-m$-form $\xi$ that depends continuously on $\sigma$ in any $C^{k}$-norm and such that

i) $\frac{d \bar{x}_{j}}{\bar{x}_{j}} \wedge \xi$ is non-singular for all $j \in \mathcal{K}$, and

ii) $d \bar{x}^{\alpha(1)} \wedge \cdots \wedge d \bar{x}^{\alpha(m)} \wedge\left(d \bar{u}_{m+1} \wedge \cdots \wedge d \bar{u}_{p} \wedge \hat{\sigma}-\xi\right)=0$.

Proof. Put $\Psi=d \bar{u}_{m+1} \wedge \cdots \wedge d \bar{u}_{p} \wedge \hat{\sigma}$ and define

$$
\tilde{\xi}=\sum_{j \in \mathcal{K}} \Psi_{j}-\sum_{\substack{i, j \in \mathcal{K} \\ i<j}} \Psi_{i j}+\cdots+(-1)^{|\mathcal{K}|-1} \Psi_{i_{1} \cdots i_{|\mathcal{K}|}},
$$

where $\Psi_{i_{1} \cdots i_{\ell}}$ means that we pull $\Psi$ back to $\left\{x_{i_{1}}=\cdots=x_{i_{\ell}}=0\right\}$ and extend trivially to $\mathbb{C}^{n}$, (i.e, $\Psi_{i_{1} \cdots i_{\ell}}=\tau^{*} \Psi$, where $\tau$ is the composition of the standard projection $\mathbb{C}^{n} \rightarrow \Lambda=\left\{x_{i_{1}}=\cdots=x_{i_{\ell}}=0\right\}$ and the inclusion $\left.\Lambda \hookrightarrow \mathbb{C}^{n}\right)$. A straight forward induction over $|\mathcal{K}|$ shows that $\xi:=\Psi-\tilde{\xi}$ satisfies i); see, e.g., [28. To see that $\xi$ satisfies ii), consider a $\Psi_{i_{1} \cdots i_{\ell}}$. Let $L \subseteq\{1, \ldots, p\}$ be the set of indices $j$ such that no $x_{i_{k}}$, $1 \leq k \leq \ell$, divides $\hat{f}_{j}$ and write $L=L^{\prime} \cup L^{\prime \prime}$, where $L^{\prime}=\{j \in L ; j \leq m\}$ and $L^{\prime \prime}=\{j \in L ; m+1 \leq j \leq p\}$.

Now, the variety $\left\{x_{i_{1}}=\cdots=x_{i_{\ell}}=0\right\}$ lies in the zero set of all the $\hat{f}_{j}$ with $j \in\{1, \ldots, p\} \backslash L$ by the definition of $L$, and moreover, it is contained in the zero set of (at least) some $\hat{f}_{\nu}$ with $p+1 \leq \nu \leq q$ since the $x_{i_{j}}$ are in $\mathcal{K}$. Thus, $\left\{x_{i_{1}}=\cdots=x_{i_{\ell}}=0\right\}$ is contained in the preimage of a subvariety of $V$ of dimension at most $n-p+|L|-1$. The form $\wedge_{j \in L} d \bar{f}_{j} \wedge \sigma$ has degree $n-p+|L|$ and so its pullback to this variety must vanish. Hence,

$$
\bigwedge_{j \in L} \widehat{d \bar{f}_{j}} \wedge \sigma=\bigwedge_{j \in L} d \hat{\bar{f}}_{j} \wedge \hat{\sigma}=\bigwedge_{i \in L^{\prime}} d \bar{x}^{\alpha(i)} \wedge \bigwedge_{j \in L^{\prime \prime}} d\left(\bar{u}_{j} \bar{x}^{\alpha(j)}\right) \wedge \hat{\sigma}
$$


has a vanishing pullback to $\left\{x_{i_{1}}=\cdots=x_{i_{\ell}}=0\right\}$. But this means that

$$
\begin{gathered}
\bar{x}^{\sum_{i \in L^{\prime \prime}} \alpha(i)} \bigwedge_{j \in L^{\prime}} d \bar{x}^{\alpha(j)} \wedge\left(\bigwedge_{k \in L^{\prime \prime}} d \bar{u}_{k} \wedge \hat{\sigma}\right)_{i_{1} \cdots i_{\ell}} \\
+\bigwedge_{\iota \in L^{\prime}} d \bar{x}^{\alpha(\iota)} \wedge \sum_{\nu \in L^{\prime \prime}} d \bar{x}^{\alpha(\nu)} \wedge \tau_{\nu}=0,
\end{gathered}
$$

where the first term arises when no differential hits $\bar{x}^{\alpha(j)}, j \in L^{\prime \prime}$. Taking the exterior product with $\bigwedge_{j \notin L^{\prime \prime}}\left(d \bar{u}_{j}\right)_{i_{1} \cdots i_{\ell}}$ we obtain

$$
\bar{x}^{\sum_{i \in L^{\prime \prime}} \alpha(i)} \bigwedge_{j \in L^{\prime}} d \bar{x}^{\alpha(j)} \wedge \Psi_{i_{1} \cdots i_{\ell}}+\bigwedge_{\iota \in L^{\prime}} d \bar{x}^{\alpha(\iota)} \wedge \sum_{\nu \in L^{\prime \prime}} d \bar{x}^{\alpha(\nu)} \wedge \tilde{\tau}_{\nu}=0 .
$$

We now multiply this equation with the exterior product of all $d \bar{x}^{\alpha(j)}$ with $j \leq m$ and $j \notin L^{\prime}$. Then we get $d \bar{x}^{\alpha(1)} \wedge \cdots \wedge d \bar{x}^{\alpha(m)}$ in front of the sum and this makes all terms under the summation sign disappear by the comment just before Lemma 9. It thus follows that

$$
\bar{x}^{\sum_{i \in L^{\prime \prime}} \alpha(i)} d \bar{x}^{\alpha(1)} \wedge \cdots \wedge d \bar{x}^{\alpha(m)} \wedge \Psi_{i_{1} \cdots i_{\ell}}=0,
$$

and since this holds everywhere we may remove the factor $\bar{x}^{\sum_{i \in L^{\prime \prime}} \alpha(i)}$ and conclude that $\xi$ has the property ii).

\section{NON-CHARACTERISTIC RESTRICTIONS}

Let $\Omega \subseteq \mathbb{R}^{k}$ be an open set, let $u \in \mathscr{D}^{\prime}(\Omega)$, and let $M \subseteq \Omega$ be a smooth submanifold. Let also $\mathcal{N}(M)$ be the subbundle of $\left.T^{*}(\Omega)\right|_{M}$ of covectors that annihilate $T(M)$. We say that $M$ is non-characteristic for $u$ if $\mathcal{N}(M) \cap W F(u)=\emptyset$, where $W F(u)$ is the wave front set of $u$. If $M$ is non-characteristic for $u$, then there is a well defined "restriction", $\left.u\right|_{M}$, of $u$ to $M$ and moreover, if $u_{\epsilon} \rightarrow u$ is any smooth regularization of $u$ and $i: M \rightarrow \Omega$ is the inclusion map, then $\left.i^{*} u_{\epsilon} \rightarrow u\right|_{M}$ is a regularization of $\left.u\right|_{M}$; see [19] Chapter VIII.

Let $\mu \in \mathbf{C H}_{V}(X)$, where $X \subseteq \mathbb{C}^{n}$ is a domain and $V$ is a pure dimensional analytic subset. Then, since $\mu$ generate a regular holonomic $\mathscr{D}_{X}$-module, [12, it follows from a deep result of Andronikof, [7], that $W F(\mu)=W F_{A}(\mu)$ is a $\mathbb{C}^{*}$-conic complex Lagrangian subset of $T^{*}(X)$. Thus, by the Morse-Sard theorem, there are "many" non-characteristic hypersurfaces for $\mu$, e.g., in appropriate coordinates, $x$, centered at an arbitrary point in $X$, all $H_{j, s}=\left\{x_{j}=s\right\}, s \in \mathbb{C}$, $0<|s|<1$, are non-characteristic for $\mu$. Now, let $f=\left(f_{1}, \ldots, f_{p}\right)$ be a holomorphic tuple defining a complete intersection in $X$ and let $R^{f}=\bar{\partial}\left(1 / f_{1}\right) \wedge \cdots \wedge \bar{\partial}\left(1 / f_{p}\right)$ be the Coleff-Herrera product.

Theorem 10. Let $Y \subseteq X$ be a complex submanifold that is noncharacteristic for $\mu$ and such that $\left.f_{1}\right|_{Y}, \ldots,\left.f_{p}\right|_{Y}$ define a complete intersection on $Y$. Then

$$
\left.R^{f}\right|_{Y}=\bar{\partial} \frac{1}{\left.f_{1}\right|_{Y}} \wedge \cdots \wedge \bar{\partial} \frac{1}{\left.f_{p}\right|_{Y}}
$$


i.e., the Coleff-Herrera product commutes with non-characteristic restrictions.

Proof. This follows immediately from our main theorem since

$$
\left.R^{f}\right|_{Y}=\lim _{\epsilon \rightarrow 0} i^{*}\left(\frac{\bar{\partial} \chi_{1}^{\epsilon} \wedge \cdots \wedge \bar{\partial} \chi_{p}^{\epsilon}}{f_{1} \cdots f_{p}}\right)=\bar{\partial} \frac{1}{\left.f_{1}\right|_{Y}} \wedge \cdots \wedge \bar{\partial} \frac{1}{\left.f_{p}\right|_{Y}}
$$

where $i: Y \rightarrow X$ is the inclusion.

Remark 11. To prove this theorem it is sufficient to use Passare regularizations. In fact, one only has to ensure that $\epsilon \rightarrow 0$ inside Passare sectors for both of the tuples $\left(f_{1}, \ldots, f_{p}\right)$ and $\left(\left.f_{1}\right|_{Y}, \ldots,\left.f_{p}\right|_{Y}\right)$.

We conclude this section with an application of Theorem 10, Formally computing the average of $I_{f}^{\varphi}(t)$ (cf., the introduction) for $t \in$ $\left\{\xi \in \mathbb{R}_{+}^{p} ; \sum_{j} \xi_{j}=\epsilon\right\}$ one obtains

$$
c_{p} \int_{|f|^{2}=\epsilon} \frac{\sum_{j}(-1)^{j-1} \bar{f}_{j} \bigwedge_{k \neq j} d \bar{f}_{k}}{|f|^{2 p}} \wedge \varphi,
$$

where $c_{p}=(-1)^{p(p-1) / 2}(p-1) ! / \sqrt{p}$. Using Hironaka's theorem and toric resolutions one can show that the limit of this integral exists and defines a Bochner-Martinelli type current $R_{B M}^{f} \in \mathbf{C H}_{f^{-1}(0)}$ that can be regularized by

$$
\bar{\partial} \chi\left(|f|^{2} / \epsilon\right) \wedge c_{p} \frac{\sum_{j}(-1)^{j-1} \bar{f}_{j} \bigwedge_{k \neq j} d \bar{f}_{k}}{|f|^{2 p}}
$$

see, e.g., [25] and 27]. If the test form $\varphi$ is $\bar{\partial}$-closed in a neighborhood of $f^{-1}(0)$, then $I_{f}^{\varphi}(t)$ is independent of sufficiently small $t$ and the formal computation above becomes rigorous. However, it is a nontrivial, but well known fact that actually $R^{f}=R_{B M}^{f}$, see [25], 2]. In fact, following [2], $R^{f}$ and $R_{B M}^{f}$ are $\nabla$-cohomologous, (see, e.g., Section 6 for the definition of the $\nabla$-operator) and, unlike the $\bar{\partial}$-operator, the $\nabla$-operator admits localizations, i.e., there are $\nabla$-closed "cut off forms". Thus, one can find a current $\mu$ with support close to $f^{-1}(0)$ such that $\nabla \mu=R^{f}-R_{B M}^{f}$ and this yields a current $\mu^{\prime}$, supported close to $f^{-1}(0)$, such that $\bar{\partial} \mu^{\prime}=R^{f}-R_{B M}^{f}$. This implies that $R^{f}=R_{B M}^{f}$ since a

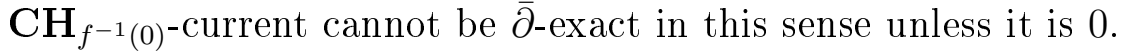

By Theorem 10 we get an independent proof of the equality $R^{f}=$ $R_{B M}^{f}$ based on induction over $n$. In the the absolute case, i.e., when $p=n$, it is easy to verify the equality by Taylor expanding the test form at the discrete set of points of $f^{-1}(0)$; we may assume that $f^{-1}(0)=0$ and then both $R^{f}$ and $R_{B M}^{f}$ are annihilated by any $\bar{x}_{j}$ and we know that they coincide on holomorphic monomials. Theorem 10 provides us with the induction step since the set of non-characteristic hyperplanes, $H_{j, s}$, is sufficiently ample. We thus get 
Corollary 12. The Bochner-Martinelli type current $R_{B M}^{f}$ is equal to the Coleff-Herrera product $R^{f}$ in the case of a complete intersection.

Remark 13. For the currents of Cauchy-Fantappiè-Leray type introduced in [1] we have the same result.

\section{Proof And extensions of Theorem 1}

Proof of Theorem 1. The proof is based on induction over $p$. The induction start, $p=0$, follows immediately from Proposition 8 (and an obvious induction over $q$ ). Now assume that Theorem 1 is true for $p=r-1 \geq 0$. By induction over $q$, what we have to show is that

$$
\left|\frac{\bar{\partial} \chi_{1}^{\epsilon} \wedge \cdots \wedge \bar{\partial} \chi_{r}^{\epsilon}}{f_{1}^{\ell_{1}} \cdots f_{r}^{\ell_{p}}} \wedge \tilde{\mu} \cdot \varphi-\bar{\partial} \frac{1}{f_{1}^{\ell_{1}}} \wedge \cdots \wedge \bar{\partial} \frac{1}{f_{r}^{\ell_{r}}} \wedge \tilde{\mu} \cdot \varphi\right| \leq C \epsilon^{\omega}\|\varphi\|_{M}
$$

where $\tilde{\mu}=\left(1 /\left(f_{r+1}^{\ell_{r+1}} \cdots f_{q}^{\ell_{q}}\right)\right) \mu \in \mathbf{C H}_{V}[* S], S:=\left\{f_{r+1} \cdots f_{q}=0\right\}$. An easy set-theoretic computation shows that $\left(f_{1}, \ldots f_{r}\right)$ defines a complete intersection on $V \cap S$ and by Proposition 5 we have $\bar{\partial} \tilde{\mu} \in \mathbf{C H}_{V \cap S}$. The left hand side of (29) can be estimated by

$$
\begin{aligned}
& \left|\frac{\bar{\partial} \chi_{\wedge}^{\epsilon} \cdots \wedge \bar{\partial} \chi_{p}^{\epsilon} \chi_{r}^{\epsilon}}{f_{1}^{\ell_{1}} \cdots \tilde{\mu} \cdot \bar{\partial} \varphi-\bar{\partial} \frac{1}{\ell_{r}}} \wedge \cdots \wedge \bar{\partial} \frac{1}{f_{1}^{\ell_{1}}} \frac{1}{f_{p}^{\ell^{\ell_{r}}}} \wedge \tilde{\mu} \cdot \bar{\partial} \varphi\right|+ \\
& \quad+\left|\frac{\bar{\partial} \chi_{1}^{\epsilon} \wedge \cdots \wedge \bar{\partial} \chi_{p}^{\epsilon} \chi_{r}^{\epsilon}}{f_{1}^{\ell_{1}} \cdots f_{r}^{\ell_{r}}} \wedge \bar{\partial} \tilde{\mu} \cdot \varphi-\bar{\partial} \frac{1}{f_{1}^{\ell_{1}}} \wedge \cdots \wedge \bar{\partial} \frac{1}{f_{p}^{\ell_{p}}} \frac{1}{f_{r}^{\ell_{r}}} \wedge \bar{\partial} \tilde{\mu} \cdot \varphi\right| .
\end{aligned}
$$

By induction, the last term can be estimated by $C \epsilon^{\omega}\|\varphi\|_{M}$. The first term can, by Proposition 8 , be estimated by $C \epsilon_{r}^{\omega}\|\bar{\partial} \varphi\|_{M}$ plus

$$
\left|\frac{\bar{\partial} \chi_{\wedge}^{\epsilon} \cdots \wedge \bar{\partial} \chi_{p}^{\epsilon}}{f_{1}^{\ell_{1}} \cdots f_{p}^{\ell_{p}}} \wedge \mu^{\prime} . \bar{\partial} \varphi-\bar{\partial} \frac{1}{f_{1}^{\ell_{1}}} \wedge \cdots \wedge \bar{\partial} \frac{1}{f_{p}^{\ell_{p}}} \wedge \mu^{\prime} . \bar{\partial} \varphi\right|,
$$

where $\mu^{\prime}=\left(1 / f_{r}^{\ell_{r}}\right) \tilde{\mu} \in \mathbf{C H}_{V}\left[* S^{\prime}\right], S^{\prime}=S \cup f_{r}^{-1}(0)$. Another "integration by parts" and the induction hypothesis finally shows that this term can be estimated by $C \epsilon^{\omega}\|\bar{\partial} \varphi\|_{M}$ and we are done.

Finally we present some extensions of our main theorem. By going through the proof one verifies the following. Let $\tilde{f}=\left(\tilde{f}_{1}, \cdots, \tilde{f}_{q}\right)$ be a holomorphic tuple such that $\left(\tilde{f}_{1}, \ldots, \tilde{f}_{p}, \tilde{f}_{j}\right)$ define a complete intersection on $V$ for all $j=p+1, \ldots, q$ and assume that $\tilde{f}^{-1}(0) \supseteq f^{-1}(0)$ and that the $\chi_{j}$ vanish to infinite order at 0 . We may then replace the $\chi_{j}\left(\left|f_{j}\right|^{2} / \epsilon_{j}\right)$ in Theorem 1 by $\chi_{j}\left(\left|\tilde{f}_{j}\right|^{2} / \epsilon_{j}\right)$ with the same conclusion; the constants $C, M$, and $\omega$ are unaffected.

Let $V$ be an analytic set of pure dimension $n$ defined in a neighborhood of $\overline{\mathbb{B}} \subset \mathbb{C}^{N}$; put $\operatorname{codim}(V)=P=N-n$. In a slightly smaller neighborhood of $\overline{\mathbb{B}}$ one can find a free resolution

$$
0 \rightarrow \mathcal{O}\left(E_{\nu}\right) \stackrel{F_{\nu}}{\longrightarrow} \cdots \stackrel{F_{2}}{\longrightarrow} \mathcal{O}\left(E_{1}\right) \stackrel{F_{1}}{\longrightarrow} \mathcal{O}\left(E_{0}\right)
$$


of the sheaf $\mathcal{O}_{\mathbb{C}^{n}} / \mathcal{J}_{V}$; the $E_{j}$ are trivial holomorphic vector bundles of ranks $r_{j}$ with $r_{0}=1$ and the $F_{j}$ are $r_{j-1} \times r_{j}$-matrices of functions holomorphic in a neighborhood of $\overline{\mathbb{B}}$. Let $V_{j}$ be the set of points $z \in \mathbb{B}$ such that $F_{j}(z)$ does not have optimal rank. These sets are analytic subsets of $\mathbb{B}$ that are independent of the choice of resolution of $\mathcal{O}_{\mathbb{C}^{n}} / \mathcal{J}_{V}$, i.e., invariants of $V$. Moreover, $V_{j} \subseteq V_{j-1} \subseteq \cdots \subseteq V_{P}=V_{P-1}=\cdots=$ $V$ and since $V$ has pure dimension in our case, Corollary 20.14 in 15 implies that for $j>P$ one has $V_{j} \subseteq V_{\text {sing }}$ and $\operatorname{codim}\left(V_{j}\right) \geq j+1$. If $k=\max \left\{j ; V_{j} \neq \emptyset\right\}$ then one can find a new resolution with $\nu=k$. Let us assume that (30) is such a minimal resolution. Let us also note that if $V$ is defined by a complete intersection, then the Koszul complex provides a minimal resolution.

Given Hermitian metrics on the $E_{j}$, Andersson-Wulcan, [6], construct a current, $R^{V}$, whose annihilator sheaf is $\mathcal{J}_{V}$. This current has the form

$$
R^{V}=R_{P}^{V}+\cdots+R_{\nu}^{V}
$$

where the $R_{j}^{V}$ are $E_{j}$-valued $(0, j)$-currents with support in $V$ and with the Standard Extension Property with respect to $V$. For some recent applications of $R^{V}$ we refer to [3] and [4]. If $\mathcal{J}_{V}$ is Cohen-Macaulay then $\nu=P$ and $R^{V}=R_{P}^{V}$ is $\bar{\partial}$-closed, in fact, $R^{V}$ is then a tuple of $\mathbf{C H}_{V}$-currents. In general, $R^{V}$ is not $\bar{\partial}$-closed but satisfies instead $\nabla_{F} R^{V}=0$, where $\nabla_{F}=\sum_{j} F_{j}-\bar{\partial}$; in the case that (30) is the Koszul complex this is the $\nabla$-operator referred to in Section 5 ,

Let $f_{1}, \ldots, f_{q} \in \mathcal{O}(\mathbb{B})$ and assume that for each $\ell \geq P$

(31) $\operatorname{codim}\left(V_{\ell} \cap\left\{f_{1}=\cdots=f_{p}=f_{j}=0\right\}\right) \geq \ell+p+1, \quad \forall j \geq p+1$.

Then one can define the product

$$
\bar{\partial} \frac{1}{f_{1}} \wedge \cdots \wedge \bar{\partial} \frac{1}{f_{p}} \frac{1}{f_{p+1}} \cdots \frac{1}{f_{q}} \wedge R^{V}
$$

by an iterative procedure similar to the one described in Section 3 and the product has the natural suggestive commutation properties, see the end of Section 2 in [5]. With our techniques one can prove

Theorem 14. Let $R^{V}$ be the current in $\mathbb{B}$ described above and let $\left(f_{1}, \ldots, f_{q}\right)$ be a tuple of holomorphic functions in $\mathbb{B}$ that satisfies (31). Then, with the notation and the hypothesis on the $\chi$-functions from Theorem 1, we have

$$
\begin{aligned}
& \mid \frac{\bar{\partial} \chi_{1}^{\epsilon} \wedge \cdots \wedge \bar{\partial} \chi_{p}^{\epsilon} \chi_{p+1}^{\epsilon} \cdots \chi_{q}^{\epsilon}}{f_{1} \cdots f_{q}} \wedge R^{V} \cdot \varphi \\
&-\bar{\partial} \frac{1}{f_{1}} \wedge \cdots \wedge \bar{\partial} \frac{1}{f_{p}} \frac{1}{f_{p+1} \cdots f_{q}} \wedge R^{V} \cdot \varphi \mid \leq C\|\varphi\|_{M} \epsilon^{\omega},
\end{aligned}
$$

for $\oplus_{j} E_{j}^{*}$-valued test forms $\varphi$ in $\mathbb{B}$. 
To prove this we need Proposition 8 with $\mu$ replaced by $R_{j}^{V}, j=$ $P, \ldots, \nu$. Then Theorem 14 follows, e.g., by a double induction over $p$ and $q$ and using that we already know that the product (32) has nice commutation properties. In the induction steps one uses that the involved $\bar{\partial}$-operators may be replaced by $-\nabla_{F}$-operators.

Let us indicate how to prove the required analogue of Proposition 8 . First of all, $R_{P}^{V}$ has an integral representation similar to (10); see, e.g., Proposition 2.1 in [4] for details. Using this, the proof of Proposition 8 goes through with $\mu$ replaced by $R_{P}^{V}$. For $R_{j}^{V}$ with $j>P$ one uses that $R_{j}^{V}=a_{j} \wedge R_{j-1}^{V}$, where $a_{j}$ is a $\operatorname{Hom}\left(E_{j-1}, E_{j}\right)$-valued $(0,1)$-form that is smooth outside $V_{j}$ and moreover has the property that in a suitable resolution $\pi: \mathcal{V} \rightarrow V, \pi^{*} a_{j}$ is a smooth $(0,1)$-form $b_{j}$ divided by a holomorphic monomial; again, see Proposition 2.1 in [4].

Now, for simplicity, consider $R_{P+1}^{V}=a_{P+1} \wedge R_{P}^{V}$ and choose such a resolution $\pi: \mathcal{V} \rightarrow V$ that, apart from having the properties in the proof of Proposition 8, also is such that the preimage of $V_{P+1}$ is a normal crossings divisor. The proof then goes through if we establish a somewhat more general division lemma, namely (see the proof of Proposition 8 for the notation):

One can replace $\Psi:=d \bar{u}_{m+1} \wedge \cdots \wedge d \bar{u}_{p} \wedge b_{P+1} \wedge \pi^{*}\left(d \bar{z}_{I}\right),|I|=n-p-1$, by a smooth form $\xi$, without affecting the integral (corresponding to (21)), such that $\left(d \bar{x}_{i} / \bar{x}_{i}\right) \wedge \xi$ is $C^{r}$-smooth for an appropriate large $r$ and all $i \in \mathcal{K}$.

This can be achieved as follows. Put

$$
\xi^{\prime}=\sum_{J \subseteq \mathcal{K}}(-1)^{|J|+1} \Psi_{J}^{r}, \quad \text { where } \quad \Psi_{J}^{r}:=\left.\sum_{j \in J, k_{j}=0}^{r+1} \frac{\partial^{|k|} \Psi}{\partial x_{J}^{k}}\right|_{x_{J}=0} \cdot \frac{x_{J}^{k}}{k !},
$$

cf., the beginning of the proof of Lemma 9, One verifies by induction that $\xi:=\Psi-\xi^{\prime}$ satisfies $\left(d \bar{x}_{i} / \bar{x}_{i}\right) \wedge \xi \in C^{r}$ for all $i \in \mathcal{K}$. Moreover, using (31) for $\ell=P+1$ and the technique of the proof of Lemma 9 one shows that $\bar{\partial} \chi_{1}^{\epsilon} \wedge \cdots \wedge \bar{\partial} \chi_{m}^{\epsilon} \wedge \xi^{\prime}=0$ so that $\Psi$ may be replaced by $\xi$ without affecting the integral.

\section{REFERENCES}

[1] M. Andersson: Residue currents and ideals of holomorphic functions. Bull. Sci. Math., 128(6) (2004), 481-512.

[2] M. Andersson: Uniqueness and factorization of Coleff-Herrera currents. Ann. Fac. Sci. Toulouse Math., to appear. Available at arXiv:0711.2440.

[3] M. Andersson, H. Samuelsson: Koppelman formulas and the $\bar{\partial}$-equation on an analytic space. Preprint, Göteborg 2008. Available at arXiv:0801.0710,

[4] M. Andersson, H. Samuelsson, J. Sznajdman: On the BrianconSkoda theorem on a singular variety. Preprint, Göteborg 2008. Available at arXiv:0806.3700.

[5] M. Andersson, E. Wulcan: Decomposition of residue currents. J. Reine Angew. Math., to appear. Available at arXiv:0710.2016. 
[6] M. Andersson, E. Wulcan: Residue currents with prescribed annihilator ideals. Ann. Sci. Ėcole Norm. Sup., 40 (2007), 985-1007.

[7] E. AndRonikof: The Kashiwara conjugation and wave-front sets of regular holonomic distributions on a complex manifold. Invent. Math., 111(1) (1993), $35-49$

[8] M. F. Aтtyah: Resolution of Singularities and Division of Distributions. Comm. Pure Appl. Math., 23 (1970), 145-150.

[9] D. Barlet, H. Maire: Asymptotic expansion of complex integrals via Mellin transforms, J. Funct. Anal., 83(2)(1989), 233-257.

[10] C. A. Berenstein, R. Gay, A. Vidras, A. Yger: Residue currents and Bezout identities. Progress in Mathematics, 114. Birkhäuser Verlag, Basel, 1993.

[11] C. A. Berenstein, R. Gay, A. Yger: Analytic continuation of currents and division problems, Forum Math., 1(1)(1989), 15-51.

[12] J.-E. BJöRK: Residues and $\mathscr{D}$-modules. The legacy of Niels Henrik Abel, 605651, Springer, Berlin, 2004.

[13] N. R. ColefF, M. E. Herrera: Les courants résiduels associés à une forme méromorphe. Lecture Notes in Mathematics, 633. Springer, Berlin, 1978.

[14] A. Dickenstein, C. Sessa: Canonical representatives in moderate cohomology. Invent. Math., 80(3) (1985), 417-434.

[15] D. Eisenbud: Commutative algebra. With a view toward algebraic geometry. Graduate Texts in Mathematics, 150. Springer-Verlag, New York, 1995.

[16] I. M. Gelfand, G. F. Shilov: Generalized functions, vol. 1., Academic Press, New York, 1964.

[17] M. E. Herrera, D. Lieberman: Residues and principal values on complex spaces. Math. Ann., 194 (1971), 259-294.

[18] H. Hironaka: Desingularization of complex-analytic varieties. Actes, Congrès Intern. Math., 1970. Tome 2, 627-631.

[19] L. HöRmander: The analysis of linear partial differential operators. I. Distribution theory and Fourier analysis. Second edition. Grundlehren der Mathematischen Wissenschaften, 256. Springer-Verlag, Berlin, 1994.

[20] P. Lelong: Propriétés métriques des variétés analytiques complexes définies par une équation. Ann. Sci. École Norm. Sup., 67(3) (1950), 393-419.

[21] M. Passare: Residues, currents, and their relation to ideals of holomorphic functions. Math. Scand., 62(1) (1988), 75-152.

[22] M. Passare: A calculus for meromorphic currents. J. Reine Angew. Math., 392 (1988), 37-56.

[23] M. Passare, A. Tsikn: Residue integrals and their Mellin transforms. Canad. J. Math., 47(5) (1995), 1037-1050.

[24] M. Passare, A. Tsikh: Defining the residue of a complete intersection. Complex analysis, harmonic analysis and applications (Bordeaux, 1995), 250-267, Pitman Res. Notes Math. Ser., 347, Longman, Harlow, 1996.

[25] M. Passare, A. Tsikh, A. YGer: Residue currents of the Bochner-Martinelli type. Publ. Mat., 44(1) (2000), 85-117.

[26] A. A. Pavlova: On some residue currents for complete intersections. (In Russian) Conf. Math. Models, Krasnojarsk, 2 (2001), 129-136.

[27] H. Samuelsson: Regularizations of products of residue and principal value currents. J. Funct. Anal, 239(2) (2006), 566-593.

[28] H. Samuelsson: Analytic continuation of residue currents. Ark. Mat., to appear. Available at: arXiv:0709.1597. 
[29] A. Yger: Formules de division et prolongement méromorphe. Séminaire d'Analyse P. Lelong-P. Dolbeault-H. Skoda, Années 1985/1986, 226-283, Lecture Notes in Math., 1295, Springer, Berlin, 1987.

J.-E. Björk, Department of Mathematics, Stockholm University, SE10691 Stockholm, Sweden

E-mail address: jeb@math.su.se

H. Samuelsson, Department of Mathematical Sciences, Division of Mathematics, University of Gothenburg and Chalmers University of Technology, SE-412 96 Göteborg, Sweden

E-mail address: hasam@math.chalmers.se 